\title{
A Warm Jupiter-sized Planet Transiting the Pre-main-sequence Star V1298 Tau
}

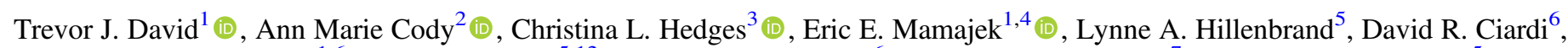
Charles A. Beichman ${ }^{1,6}$, Erik A. Petigura ${ }^{5,12}$ (1), Benjamin J. Fulton ${ }^{6}$ (i), Howard T. Isaacson ${ }^{7}$ (i), Andrew W. Howard ${ }^{5}$ (10), Jonathan Gagné $^{8,13}$ (1) , Nicholas K. Saunders ${ }^{2}$, Luisa M. Rebull ${ }^{9}$ (1) , John R. Stauffer ${ }^{10}$ (1) , Gautam Vasisht ${ }^{1}$, and Sasha Hinkley ${ }^{11}$ (1)

${ }^{1}$ Jet Propulsion Laboratory, California Institute of Technology, 4800 Oak Grove Drive, Pasadena, CA 91109, USA; trevor.j.david@jpl.nasa.gov ${ }^{2}$ NASA Ames Research Center, Moffett Field, CA 94035, USA

${ }^{3}$ Bay Area Environmental Research Institute, P.O. Box 25, Moffett Field, CA 94035, USA

${ }^{4}$ Department of Physics \& Astronomy, University of Rochester, Rochester, NY 14627, USA

${ }^{5}$ Department of Astronomy, California Institute of Technology, Pasadena, CA 91125, USA

${ }^{6}$ Caltech/IPAC-NASA Exoplanet Science Institute, Pasadena, CA 91125, USA

${ }^{7}$ Astronomy Department, University of California, Berkeley, CA 94720, USA

${ }^{8}$ Institut de Recherche sur les Exoplanètes, Département de Physique, Université de Montréal, Montréal QC, H3C 3J7, Canada

${ }^{9}$ Caltech/IPAC-IRSA, Pasadena, CA 91125, USA

${ }^{10}$ Caltech/IPAC-SSC, Pasadena, CA 91125, USA

${ }^{11}$ University of Exeter, Physics Department, Stocker Road, Exeter EX4 4QL, UK

Received 2019 February 25; revised 2019 June 6; accepted 2019 June 10; published 2019 July 25

\begin{abstract}
We report the detection of V1298 Tau b, a warm Jupiter-sized planet $\left(R_{\mathrm{P}}=0.91 \pm 0.05 R_{\mathrm{Jup}}, P=24.1\right.$ days $)$ transiting a young solar analog with an estimated age of 23 Myr. The star and its planet belong to Group 29, a young association in the foreground of the Taurus-Auriga star-forming region. While hot Jupiters have been previously reported around young stars, those planets are non-transiting and near-term atmospheric characterization is not feasible. The V1298 Tau system is a compelling target for follow-up study through transmission spectroscopy and Doppler tomography owing to the transit depth $(0.5 \%)$, host star brightness $\left(K_{s}=8.1 \mathrm{mag}\right)$, and rapid stellar rotation $\left(v \sin i=23 \mathrm{~km} \mathrm{~s}^{-1}\right)$. Although the planet is Jupiter-sized, its mass is currently unknown due to high-amplitude radial velocity jitter. Nevertheless, V1298 Tau b may help constrain formation scenarios for at least one class of close-in exoplanets, providing a window into the nascent evolution of planetary interiors and atmospheres.
\end{abstract}

Key words: open clusters and associations: individual (Group 29, Taurus-Auriga) - planets and satellites: gaseous planets - planets and satellites: physical evolution - stars: individual (V1298 Tau) - stars: pre-main sequence

\section{Introduction}

The properties and demographics of young exoplanets may potentially reveal valuable insights into the physics of planet formation. For example, theoretical models predict that the radii of super-Earths and mini-Neptunes were significantly larger at ages $\lesssim 100 \mathrm{Myr}$ (Owen \& Wu 2013). The valley observed in the radius distribution of small planets (Fulton et al. 2017; Fulton \& Petigura 2018) is then expected to form gradually through some combination of photoevaporation (Lopez \& Fortney 2013; Owen \& Wu 2013), planetary impacts (Schlichting et al. 2015), and core-envelope interactions (Ginzburg et al. 2018; Gupta \& Schlichting 2019). All of the aforementioned processes result in net atmospheric loss, but it is not clear which mechanism dominates.

For giant planets, the post-formation radii, temperatures, and luminosities may help to constrain how planets accrete gaseous envelopes and perhaps whether or not they possess rocky cores (e.g., Burrows et al. 1997; Baraffe et al. 2003; Fortney et al. 2007; Marley et al. 2007; Fortney \& Nettelmann 2010; Spiegel \& Burrows 2012; Mordasini 2013; Mordasini et al. 2017).

Discerning which physical mechanisms are important to the evolution of exoplanets, and which are not, requires studying planetary systems of a variety of ages. For this reason, the task of identifying exoplanets in stellar associations with well-defined ages has attracted significant attention lately (Quinn et al. 2012, 2014;

\footnotetext{
${ }^{12}$ NASA Hubble Fellow.

${ }^{13}$ Banting Fellow.
}

Libralato et al. 2016; Mann et al. 2016a, 2017, 2018; Obermeier et al. 2016; Pepper et al. 2017; Ciardi et al. 2018; Curtis et al. 2018; Livingston et al. 2018, 2019; Rizzuto et al. 2018; Vanderburg et al. 2018).

At the youngest ages $(<100 \mathrm{Myr})$, when observed exoplanet properties might place the strongest constraints on formation theories, there are only a small number of known exoplanets; several young giant planets have been discovered via direct imaging (see Bowler 2016 for a review) while there are only five validated or candidate close-in exoplanets with securely measured ages. Three of the close-in planets are hot Jupiters detected through radial velocity monitoring of $\mathrm{T}$ Tauri stars (Donati et al. 2016; Johns-Krull et al. 2016; Yu et al. 2017), one is a candidate transiting hot Jupiter of controversial nature (van Eyken et al. 2012; Yu et al. 2015), and the other is a Neptune-sized planet transiting a pre-main-sequence star (David et al. 2016; Mann et al. 2016b).

NASA's Kepler Space Telescope observed thousands of young stars during its extended $K 2$ mission (Howell et al. 2014). For most of these stars, $K 2$ provided time-series photometry with unprecedented precision, cadence, and duration. Using astrometry from the second data release of the Gaia mission (Gaia Collaboration et al. 2018) and a Bayesian membership algorithm (Gagné et al. 2018), we searched the entire $K 2$ source catalog for high-probability members of young moving groups and stellar associations. Our search yielded 432 candidate members of the Taurus-Auriga (Tau-Aur) starforming region, including V1298 Tau. In an automated search 

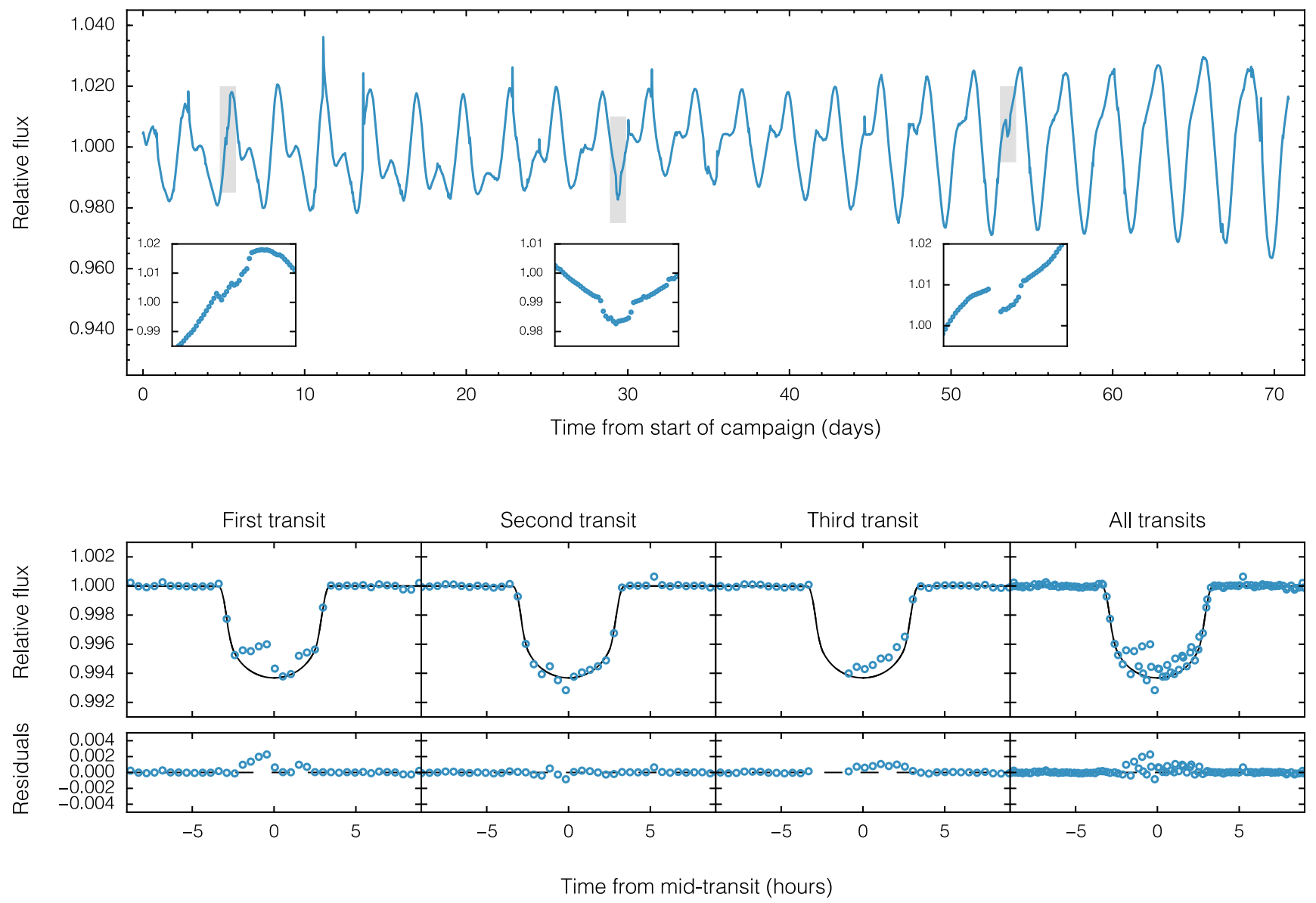

Figure 1. $K 2$ time-series photometry of V1298 Tau. Top: systematics-corrected $K 2$ photometry of V1298 Tau from the EVEREST 2.0 pipeline. Inset panels show daylong windows around individual transits, also indicated by the gray shaded regions. Bottom: folded and individual transits of V1298 Tau b (points) with a transit model fit (solid line) found from excluding anomalous observations. Residuals of this fit (Fit 1, as described in Section 3.4) are shown below each panel. Observations missing from the ingress of the third transit are due to an interruption in data acquisition which affected all targets in Campaign 4.

for periodic transits or eclipses within the the $K 2$ photometry for these candidate members we detected $0.5 \%$ dimming events which last $6.4 \mathrm{hr}$ and repeat every 24.1 days within the light curve of V1298 Tau (Figure 1). Follow-on observations confirm the periodic transits are most probably due to a Jovian-sized planet orbiting at a separation of 0.17 au from V1298 Tau.

In Section 2 we describe the $K 2$ time-series photometry from which the planet V1298 Tau b was detected and the follow-on observations used to validate the planet. Our analysis of these observations and assessment of false-positive scenarios is described in Section 3. We place the planet V1298 Tau b in context in Section 4, and present our conclusions in Section 5.

\section{Observations and Characterization of the Data}

\subsection{K2 Time-series Photometry}

Kepler observed V1298 Tau during Campaign 4 of the $K 2$ mission (Howell et al. 2014), between 2015 February 7 and 2015 April 23 UTC. The star was included in the following Guest Observer programs: GO4020 (Stello), GO4057 (Prša), GO4060 (Coughlin), GO4090 (Caldwell), GO4092 (Brown), and GO4104 (Dragomir). Telescope pointing drift combined with intra-pixel detector sensitivity variations imprint systematic artifacts upon $K 2$ time series photometry. We corrected for these systematic effects using the EVEREST 2.0 pipeline (Luger et al. 2018), which employs a variant of the pixel-level decorrelation (PLD) method (Deming et al. 2013). We also confirmed the transits are present in the raw uncorrected photometry using the interactive LIGHTKURVE tool (Barentsen et al. 2019), as well as in independent reductions of the data using the K2SC (Aigrain et al. 2016) and K2PHOT (Petigura et al. 2018) pipelines.

The $K 2$ time-series photometry is characterized by quasiperiodic variations with a peak-to-trough amplitude of $\sim 6 \%$ and a period of $2.851 \pm 0.050$ days (Section 3.3), values typical of similarly young stars (Rebull et al. 2018). We attribute this variability to stellar rotation and a nonaxisymmetric distribution of spots on the stellar surface. The photometry also reveal several flares over the 70.8 day observing baseline, confirming that the star is magnetically active. Prior to fitting transit models to the photometry, we removed the intrinsic stellar variability through Gaussian process (GP) regression. For the GP we used a Matérn-3/2 kernel with an additional white noise term, using a white noise amplitude of $243 \mathrm{ppm}$, red noise amplitude of $1.215 \times 10^{5}$ counts, and red noise timescale of 29.17 days. The transits were masked prior to both the PLD detrending and the final GP regression to prevent under- or over-fitting the transit signal.

From inspection of systematics-corrected light curves using both the EVEREST 2.0 and K2SC pipelines we found that there are several outlying or missing in-transit measurements (Figure 2). To investigate the potential roles of known systematic effects in causing these outliers, we examined the 

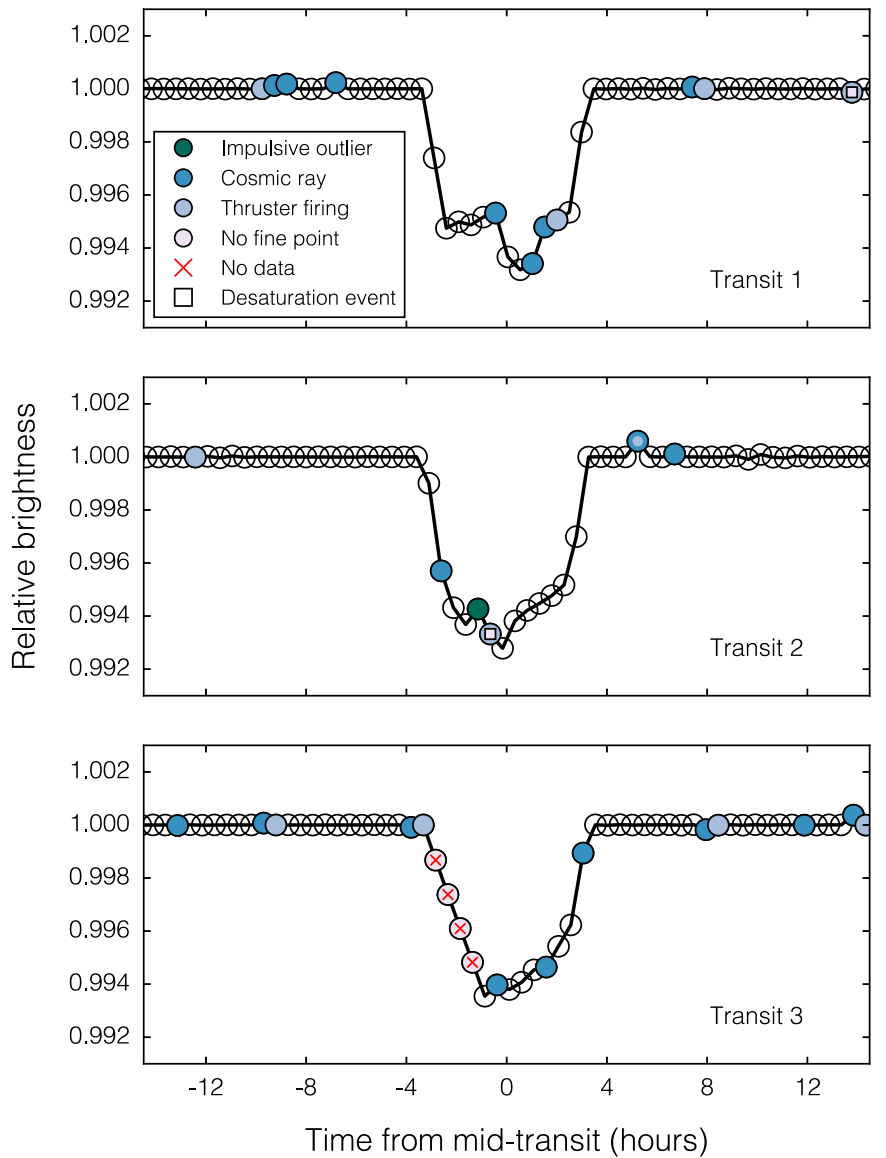
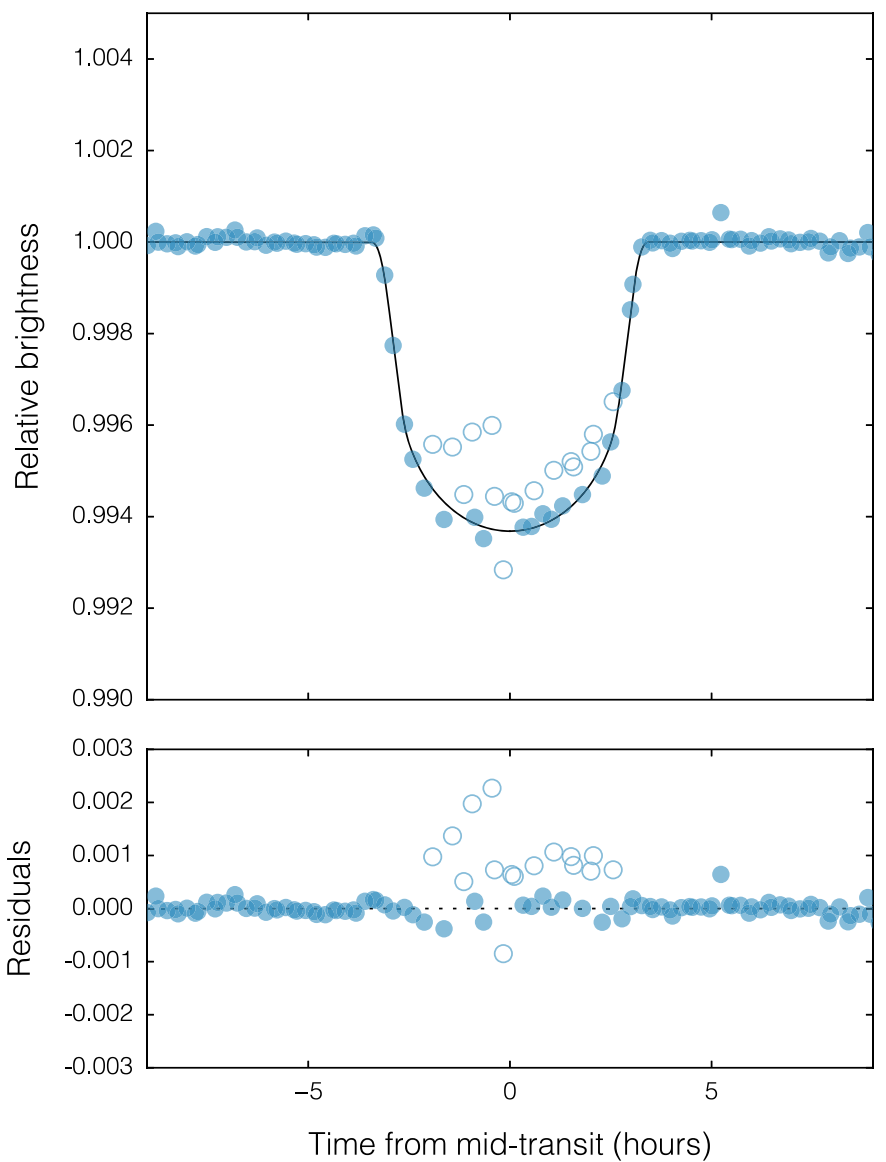

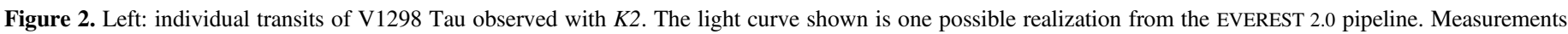

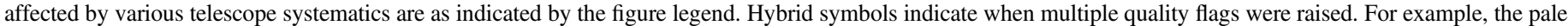

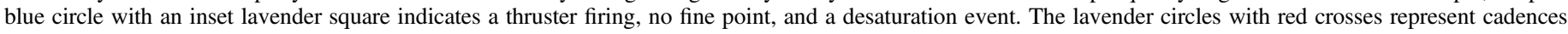

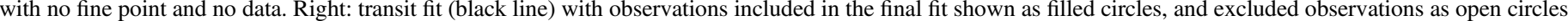
(Fit 1 is shown, as described in Section 3.4).

quality flags ${ }^{14}$ for each in-transit observation. There are six unique data quality flags raised during the in-transit observations of V1298 Tau. We summarize the flag meanings here.

1. Impulsive outlier. The Kepler presearch data conditioning (PDC) pipeline (Smith et al. 2012; Stumpe et al. 2012) identifies outliers before cotrending. These "impulsive outliers" may be astrophysical (such as deep transits or cosmic ray hits) or systematic.

2. Cosmic ray. This quality flag indicates that a cosmic ray impinged on at least one of the pixels contained in the photometric aperture. Cosmic rays can affect pixels in the short term (depositing excess charge which is cleared on the subsequent destructive readout), medium term (temporarily changing a pixel's sensitivity), or long term (sometimes changing a pixel's sensitivity permanently).

3. Thruster firing. During the $K 2$ mission, spacecraft thrusters were fired every $\sim 6 \mathrm{hr}$ to mitigate pointing drift. This strategy constrains the excursions of a star on the detector, but introduces sawtooth-like systematics in the photometry due to intra-pixel sensitivity variations. Observations acquired during a thruster firing may thus

\footnotetext{
${ }^{14}$ Quality flag meanings are summarized in Table 2.3 of the Kepler archive manual (https://archive.stsci.edu/kepler/manuals/archive_manual.pdf), discussed in detail in the Kepler Data Characteristics Manual (https://archive. stsci.edu/kepler/manuals/Data_Characteristics.pdf), and may be interpreted with software available at https://gist.github.com/barentsen/.
}

appear as outliers, depending on how the data are detrended.

4. No fine point. Occasionally the Kepler spacecraft loses fine pointing control. A degradation of pointing precision may be accompanied by increased image motion and thus reduced photometric precision.

5. No data. As implied, this quality flag indicates the spacecraft was not collecting data.

6. Desaturation event. When angular momentum builds up in the reaction wheels (due to solar radiation torques), a thruster firing is required to "desaturate" the reaction wheels and keep their speeds within operating limits. During the $K 2$ mission, this quality flag was also used to indicate a "resaturation event," in which thrusters were fired to keep reaction wheels safely away from zero angular velocity. Fine pointing control is not maintained during desaturation/resaturation events, and so the subsequent motion of the star on the detector can lead to a loss in photometric precision. Desaturation/resaturation events can affect a single long-cadence observation.

For V1298 Tau, most notably, observations of the third transit ingress (cadences 106358 through 106361), are missing due to the fact that the Kepler spacecraft was not in fine pointing mode and did not acquire data. The outlying observations during the first transit, which are present in both the EVEREST 2.0 and K2SC light curves, are not as easily 
explained. Transit profile distortions may be systematic or astrophysical in nature, and possibly the result of both effects. Quality flags from the $K 2$ photometry indicate that some observations taken during the first transit were affected by cosmic rays or thruster firings (dark blue and pale blue circles, respectively, in Figure 2). However, observations closely spaced in time which also appear to be outliers were not affected by such systematics, and thruster firings in particular are naturally detrended with the PLD approach of the EVEREST 2.0 pipeline. It is possible that the outliers are instead astrophysical in nature, arising from the planet occulting active regions on the stellar surface. The transit depth is reduced when the planet crosses a dark starspot and increased if the planet crosses a bright plage or flaring region. With only three transits, we cannot conclusively attribute any outliers to spot-crossing events, but the light curve of V1298 Tau is indeed suggestive of a non-axisymmetric spotted stellar surface.

\subsection{High-resolution Imaging}

We observed V1298 Tau with infrared high-resolution adaptive optics (AO) imaging at Keck Observatory. The Keck Observatory observations were made with the NIRC2 instrument on Keck II behind the natural guide star AO system. The observations were made on 2018 November 22 UT in the standard three-point dither pattern that is used with NIRC2 to avoid the left lower quadrant of the detector which is typically noisier than the other three quadrants. The dither pattern step size was $3^{\prime \prime}$ and was repeated twice, with each dither offset from the previous dither by $0 . " 5$.

The observations were made in the narrow-band $\mathrm{Br}-\gamma$ filter $\left(\lambda_{o}=2.1686 ; \Delta \lambda=0.0326 \mu \mathrm{m}\right)$ with an integration time of $1.25 \mathrm{~s}$ with one coadd per frame for a total of $11.25 \mathrm{~s}$ on target and in $J$-cont $\left(\lambda_{o}=1.2132 ; \Delta \lambda=0.0198 \mu \mathrm{m}\right)$ with an integration time of $5 \mathrm{~s}$ with one coadd per frame for a total of $45 \mathrm{~s}$ on target. The camera was in the narrow-angle mode with a full field of view of $\sim 10^{\prime \prime}$ and a pixel scale of approximately 9.9442 mas per pixel. The Keck AO observations show no additional stellar companions were detected to within a resolution $\sim 0$." 05 full width at half maximum (FWHM).

The sensitivities of the final combined AO image were determined by injecting simulated sources azimuthally around the primary target every $45^{\circ}$ at separations of integer multiples of the central source's FWHM (Furlan et al. 2017). The brightness of each injected source was scaled until standard aperture photometry detected it with $5 \sigma$ significance. The resulting brightness of the injected sources relative to the target set the contrast limits at that injection location. The final $5 \sigma$ limit at each separation was determined from the average of all of the determined limits at that separation; the uncertainty on the $5 \sigma$ limit was set by the root mean square (rms) dispersion of the azimuthal slices at a given radial distance.

\subsection{Spectroscopic Observations and Radial Velocities}

We observed V1298 Tau on nine nights between 2018 November 3 and 2019 January 26 UTC using the High Resolution Spectrograph (HIRES) on the Keck I telescope (Vogt et al. 1994). We first acquired a spectrum for characterization purposes on 2018 November 3, before commencing precision radial velocity (PRV) monitoring with the iodine cell. Our characterization spectrum had a resolution
Table 1

Keck/HIRES Precision Radial Velocities of V1298 Tau

\begin{tabular}{lcccc}
\hline \hline Observation & UTC Date & JD & $\begin{array}{c}\mathrm{RV} \\
\left(\mathrm{m} \mathrm{s}^{-1}\right)\end{array}$ & $\begin{array}{c}\sigma_{\mathrm{RV}} \\
\left(\mathrm{m} \mathrm{s}^{-1}\right)\end{array}$ \\
\hline rj310.68 & 2018 Nov 16 & 2458438.94547 & +459.27 & 6.23 \\
rj311.73 & 2018 Nov 21 & 2458443.81966 & -233.24 & 4.73 \\
rj311.113 & 2018 Nov 21 & 2458443.95650 & -67.81 & 3.91 \\
rj311.114 & 2018 Nov 21 & 2458443.96733 & -56.12 & 4.26 \\
rj311.115 & 2018 Nov 21 & 2458443.97835 & -64.60 & 4.00 \\
rj311.172 & 2018 Nov 21 & 2458444.15574 & -37.38 & 5.58 \\
rj314.102 & 2018 Dec 24 & 2458476.80809 & +147.66 & 8.48 \\
rj315.63 & 2018 Dec 26 & 2458479.00517 & +14.88 & 7.80 \\
rj316.90 & 2019 Jan 7 & 2458490.79036 & +191.34 & 8.39 \\
rj316.376 & 2019 Jan 8 & 2458491.75402 & -101.69 & 7.34 \\
rj317.81 & 2019 Jan 25 & 2458508.91881 & -136.91 & 8.72 \\
rj317.398 & 2019 Jan 26 & 2458509.78912 & +127.77 & 8.76 \\
\hline & & & &
\end{tabular}

Table 2

Properties of V1298 Tau

\begin{tabular}{lc}
\hline \hline Parameter & Value \\
\hline Designations & K2-309 \\
& EPIC 210818897
\end{tabular}

[WKS96] 4

R.A. (J2000.0)

RX J0405.3+2009 $04^{\mathrm{h}} 05^{\mathrm{m}} 19^{\mathrm{s}} \cdot 6$

Decl. (J2000.0)

Parallax, $\varpi$ (mas) $+20^{\circ} 09^{\prime} 25^{\prime \prime} 6$

$9.214 \pm 0.059$

$5.23 \pm 0.13$

Proper motion R.A., $\mu_{\alpha}\left(\right.$ mas $\left.\mathrm{yr}^{-1}\right)$

Proper motion decl., $\mu_{\delta}\left(\right.$ mas $\left.\mathrm{yr}^{-1}\right)$

Distance, $d(\mathrm{pc})$

Spectral type

Stellar age, $\tau_{\star}(\mathrm{Myr})$

Stellar mass, $M_{\star}\left(M_{\odot}\right)$

Stellar radius, $R_{\star}\left(R_{\odot}\right)$

Effective temperature, $T_{\text {eff }}(\mathrm{K})$

Luminosity, $L_{\star}\left(L_{\odot}\right)$

Mean stellar density, $\rho_{\star}\left(\mathrm{g} \mathrm{cm}^{-3}\right)$

Surface gravity, $\log g_{\star}(\mathrm{dex})$

Stellar rotation period, $P_{\text {rot }}(\mathrm{d})$

Projected rotational velocity, $v \sin i\left(\mathrm{~km} \mathrm{~s}^{-1}\right)$

Barycentric radial velocity, $\gamma\left(\mathrm{km} \mathrm{s}^{-1}\right)$

Li I $6708 \AA$ equivalent width $(\mathrm{m} \AA)$

$(B-V)$ color excess, $E(B-V)(\mathrm{mag})$

$6.077 \pm 0.04$

$108.5 \pm 0.7$

K0-K1.5

$23 \pm 4$

$1.10 \pm 0.05$

$1.305 \pm 0.070$

$4970 \pm 120$

$0.934 \pm 0.044$

$0.697 \pm 0.075$

$4.246 \pm 0.034$

$2.865 \pm 0.012$

$23 \pm 2$

$16.15 \pm 0.38$

380

$0.024 \pm 0.015$

$J$-band extinction, $A_{J}(\mathrm{mag})$

$0.019 \pm 0.010$

Note. Astrometric parameters originate from Gaia DR2.

of $R \approx 36,000$ between $\sim 4800$ and $9200 \AA$. For the PRV observations, a template spectrum with resolution of $R \approx 80,000$ was obtained for use with the California Planet Survey RV pipeline (Howard et al. 2010a). The PRV observations themselves had a resolution of $R \approx 50,000$ between $\sim 3600$ and $8000 \AA$. The PRVs for V1298 Tau are presented in Table 1. From those data we measured the optical radial velocity jitter to be $\sigma_{\mathrm{RV}}=216,71$, and $5 \mathrm{~m} \mathrm{~s}^{-1}$ over 5.2 days, $10 \mathrm{hr}$, and $30 \mathrm{~min}$, respectively. While a measurement of the planet mass is precluded by the large stellar variability, we derive an upper limit in Section 3.12.

For the first epoch of spectroscopic observations, the radial velocity was derived by cross-correlating the spectrum with a radial velocity standard (Table 2). For this measurement we used the G2 type standard HD 3765 (Nidever et al. 2002), which we 
observed with HIRES on the same night and in the same spectrograph configuration. Uncertainty was quantified from the dispersion among measurements relative to different standards, and over 24 different spectral orders. The sky-projected rotational velocity was also estimated from this spectrum by artificially broadening the spectral standard Gl 651 (SpT = G8) using the standard Gray broadening profile (Gray 2005) with $\epsilon=0.6$. Velocities between 9 and $50 \mathrm{~km} \mathrm{~s}^{-1}$ were sampled and the best-fit value for $v \sin i$ was determined by minimizing residuals, suggesting $23 \pm 2 \mathrm{~km} \mathrm{~s}^{-1}$.

\section{Analysis}

\subsection{Association Membership and Kinematics}

V1298 Tau was first proposed as a young star and candidate Tau-Aur member based on a detection of its X-ray emission from the ROSAT All-sky Survey (Wichmann et al. 1996). The star's relative youth (age $\lesssim 100 \mathrm{Myr}$ ) was then verified on the basis of strong lithium $6708 \AA$ absorption (Wichmann et al. 2000). Tau-Aur, at $\sim 3 \mathrm{Myr}$, contains both classical T Tauri stars in the process of forming planets as well as weak-line $\mathrm{T}$ Tauri stars lacking accretion signatures. V1298 Tau, an early K-type star which has no significant infrared excess and exhibits $\mathrm{H} \alpha$ in absorption, could fit into the latter category.

Alternatively, while V1298 Tau is young, it may be significantly older than $3 \mathrm{Myr}$. The existence of an older and more spatially distributed pre-main-sequence population in the vicinity of Taurus has been recognized (e.g., Hartmann et al. 1991; Slesnick et al. 2006; Kraus et al. 2017; Zhang et al. 2018), but a recent kinematic analysis enabled by Gaia suggests the older population is physically unrelated to the Tau-Aur association (Luhman 2018). Many of the more spatially distributed candidate young stars likely belong to a newly identified, older association preliminarily named Group 29 (Luhman 2018), to which V1298 Tau was proposed to belong (Oh et al. 2017).

The Gaia astrometry and barycentric radial velocity of V1298 Tau were used to estimate the star's probability of membership to the Tau-Aur star-forming region. Using the BANYAN $\Sigma$ classification tool (Gagné et al. 2018), we found that V1298 Tau has a $99.8 \%$ probability of belonging to Taurus and a $0.2 \%$ probability of being a field star. However, BANYAN $\Sigma$ utilizes an inclusive model for Tau-Aur and does not yet take into account the aforementioned analysis of kinematic substructure in the association.

From the Hertzsprung-Russell diagram position (Figure 3), stellar kinematics (Figure 4), and spatial location relative to the Taurus molecular clouds (Figure 4), we conclude that V1298 $\mathrm{Tau}$ is a member of Group 29, an older association in the foreground of Tau-Aur. The age of Group 29 is estimated to be less than 45 Myr by comparison with known moving groups in a color-absolute magnitude diagram (Luhman 2018; see also Figure 5). We note, however, that the precise age and substructure of this group has not been fully explored. A comparison of $\mathrm{Li}$ I $6708 \AA$ equivalent widths among young stars compiled from the literature ${ }^{15}$ supports an upper limit of 45 Myr for the age of V1298 Tau (Figure 6).

\footnotetext{
$15 \mathrm{Li} 6708 \AA$ equivalent widths and temperatures/spectral types were compiled from Soderblom et al. (1993), Jones et al. (1996), Barrado y Navascués et al. (2001), Randich et al. (2001), Mentuch et al. (2008), Yee \& Jensen (2010), Malo et al. (2013), Jeffries et al. (2013), Binks \& Jeffries (2014), Kraus et al. (2014, 2017), and Bouvier et al. (2018).
}
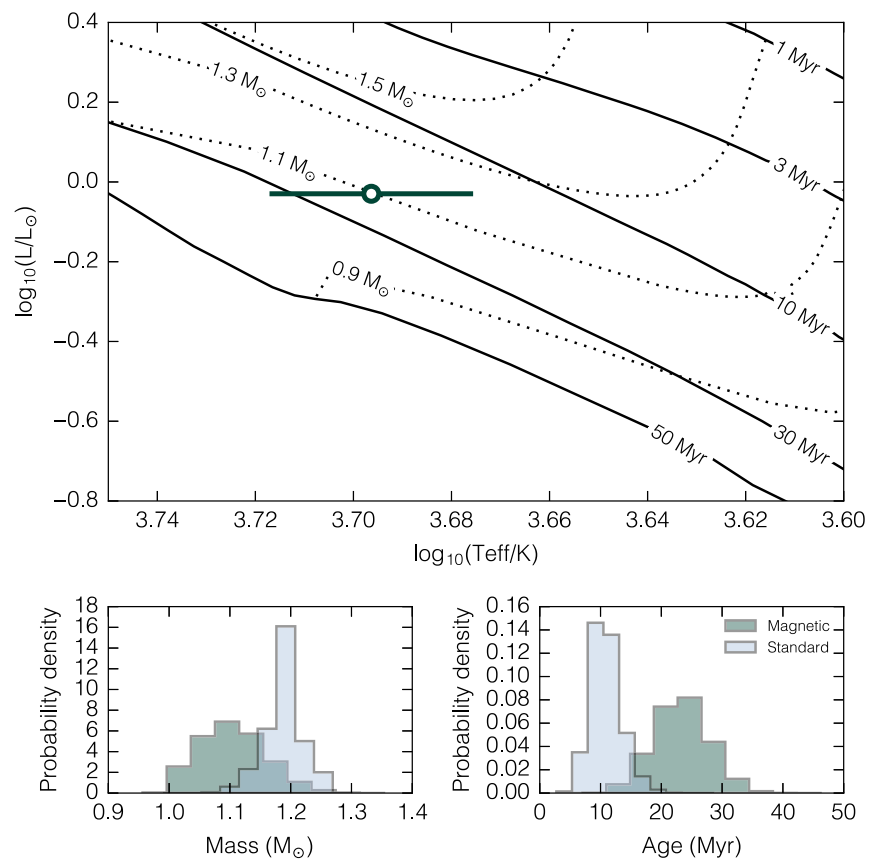

Figure 3. Top: V1298 Tau (blue point) in a theoretical Hertzsprung-Russell diagram. Isochrones (solid lines) and mass tracks (dotted lines) from Dartmouth models including the effects of magnetic fields (Feiden 2016) are shown. Bottom: mass and age distributions from Monte Carlo simulations for V1298 Tau according to magnetic (green) and standard (blue; Dotter et al. 2008) Dartmouth evolutionary models. Given the precise parallax and near-infrared photometry, the uncertainties in mass and age are dominated by the error in $T_{\text {eff }}$ and model systematics.

\subsection{Stellar Parameters}

We determined the stellar effective temperature, luminosity, and radius from 2MASS near-infrared photometry, the trigonometric parallax, and empirical relations for pre-mainsequence stars (Pecaut \& Mamajek 2013). From a threedimensional map of the local interstellar medium, we determined the color excess along the line of sight to V1298 Tau to be $E(B-V)=0.024 \pm 0.015$ mag (Lallement et al. 2014; Capitanio et al. 2017). Assuming an extinction law (Yuan et al. 2013), we determined the $J$-band extinction to be $A_{J}=0.019 \pm 0.010 \mathrm{mag}$ and the star's intrinsic $J-K_{s}$ color $\left(J-K_{s}\right)_{0}=0.582 \pm 0.032 \mathrm{mag}$. From the intrinsic $\left(J-K_{s}\right)_{0}$ color and linear interpolation between empirical pre-mainsequence relations, we determined the effective temperature and the appropriate $J$-band bolometric correction. Using the extinction-corrected $J$-band magnitude we then calculated the bolometric luminosity. From the luminosity and effective temperature, we determined the stellar radius from the StefanBoltzmann law. Our adopted temperature is consistent with previous determinations, which range from 4920 to $5080 \mathrm{~K}$ (Wichmann et al. 2000; Palla \& Stahler 2002; Wahhaj et al. 2010; Davies et al. 2014). The spectral type has been reported as K1 (Wichmann et al. 1996) and K1.5 (Kraus et al. 2017), and we find the HIRES spectrum to be most consistent with K0. The mass, estimated below, suggests V1298 Tau will evolve to become a late F-type or early G-type mainsequence star.

We used stellar evolution models to estimate the mass and age of V1298 Tau in a theoretical Hertzsprung-Russell diagram. The pre-main-sequence phase of evolution is particularly uncertain in theoretical models due to a dearth of 


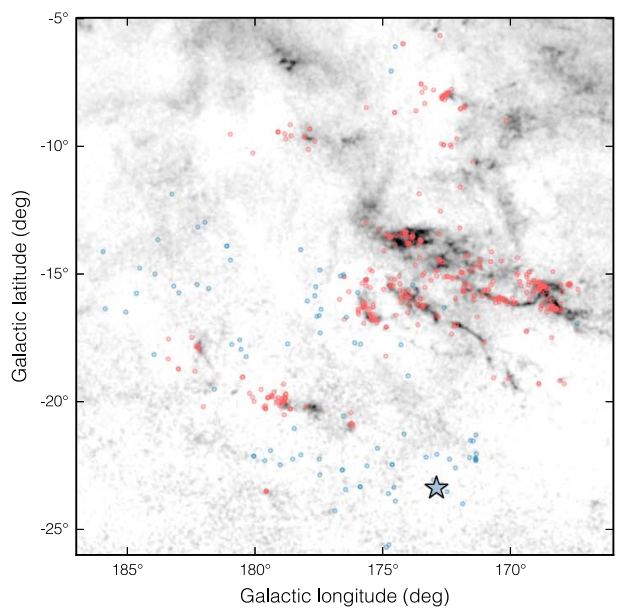

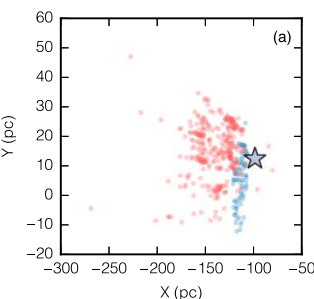

$x(\mathrm{pc})$

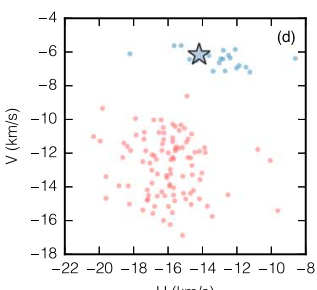

$\mathrm{U}(\mathrm{km} / \mathrm{s})$

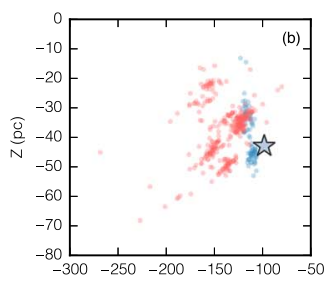

$x(\mathrm{pc})$

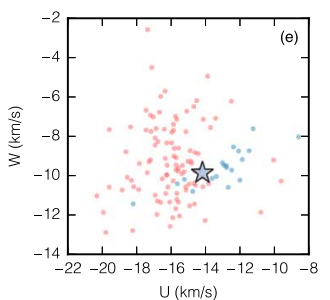

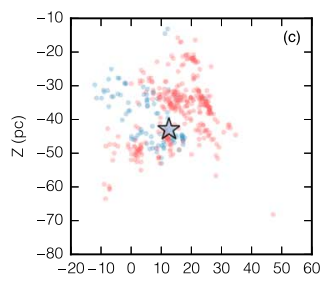

$Y(p c)$

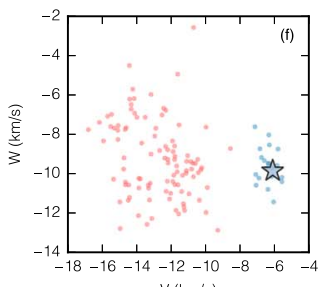

$\mathrm{V}(\mathrm{km} / \mathrm{s})$

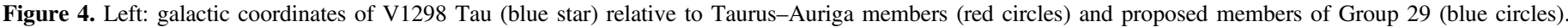

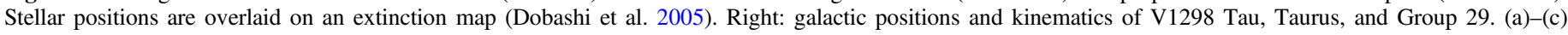

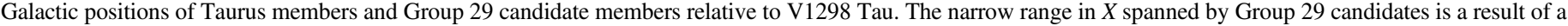

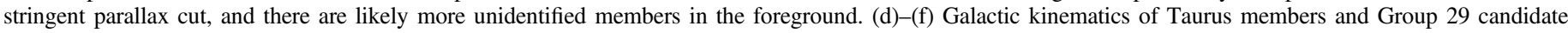
members relative to V1298 Tau.

calibrators. One major uncertainty regards the importance of magnetic fields. To estimate the magnitude of the model uncertainty for V1298 Tau we present two determinations of mass and age using models that neglect and account for magnetic fields (Dotter et al. 2008; Feiden 2016). Standard models produce a mass and age of $M_{*}=1.19 \pm 0.03 M_{\odot}$ and $\tau=11 \pm 3 \mathrm{Myr}$, respectively. By comparison, magnetic models produce a mass of $M_{*}=1.10 \pm 0.05 M_{\odot}$ and an age of $\tau=23 \pm 4 \mathrm{Myr}$, which we ultimately adopt (Figure 3). Uncertainties in all derived stellar parameters were calculated from Monte Carlo simulations modeling input parameters as normal distributions with widths corresponding to the errors in the photometry, parallax, and extinction. The derived stellar parameters are summarized in Table 2.

As mentioned earlier, V1298 Tau lacks either spectroscopic accretion signatures or an infrared excess out to $24 \mu \mathrm{m}$, with an upper limit to the fractional disk luminosity of $L_{\text {disk }} / L_{\star}<1.5 \times 10^{-4}$ (Wahhaj et al. 2010). The star's spectral energy distribution is well-described by a photosphere with excess emission in the far and near ultraviolet (see Section 3.3). The lack of any significant circumstellar disk is consistent with the age we infer for V1298 Tau.

\subsection{Stellar Rotation and Activity}

We infer a stellar rotation period of $P_{\text {rot }}=2.851 \pm 0.050$ days from a Lomb-Scargle periodogram (Lomb 1976; Scargle 1982) of the K2 time series photometry (Figure 7). The period and uncertainty were determined from the mean and the half-width at half-maximum of a Gaussian fit to the periodogram peak, respectively. An autocorrelation function (ACF) of the $K 2$ light curve suggests a rotation period of $P_{\text {rot }}=2.8605 \pm 0.0082$ days, consistent within $1 \sigma$. In this case the period is determined from the slope of a linear fit to the first four peaks of the ACF, with the uncertainty determined from the rms of the fit residuals. The period we report is in agreement with a previously published value (Grankin et al. 2007), and consistent with the period distribution among similarly young stars (Figure 8). The uncertainties we quote for the rotation period reflect a measurement error and do not account for differential rotation, which can be as large as
$0.2 \mathrm{rad} \mathrm{day}^{-1}$ for pre-main-sequence stars (Waite et al. 2011). The amplitude of brightness modulations is seen to evolve throughout the $K 2$ observation period. Such an effect may be observed when two signals with different periods give rise to a beat pattern, which may be due to surface differential rotation, star spot emergence and decay, or two stars contained within the photometric aperture. The rotation period was also measured through GP regression, as described in Section 3.5, and found to be $P_{\text {rot }}=2.865 \pm 0.012$ days, which we ultimately adopt.

V1298 Tau exhibits excess ultraviolet emission, a common characteristic of similarly young stars. Using data from the GALEX mission, Findeisen \& Hillenbrand (2010) found a farultraviolet excess of $3.4 \pm 0.7 \mathrm{mag}$ (at $4.7 \sigma$ significance) and a near-ultraviolet excess of $0.8 \pm 0.3 \mathrm{mag}(2.2 \sigma)$. The level of ultraviolet excess exhibited by V1298 Tau is consistent with members of a similar color in the Lower Centaurus-Crux and Upper Centaurus-Lupus associations (15-25 Myr), while lower than members of the Upper Scorpius OB association (5-10 Myr) and higher than the Kepler sample (Olmedo et al. 2015, see Figure 9).

From the ROSAT X-ray flux $\left(f_{\mathrm{X}}=1.64 \times 10^{-15} \mathrm{~W} \mathrm{~m}^{-2}\right)$, a published MEKAL plasma fit, and hydrogren column density (Boller et al. 2016), we estimate an X-ray luminosity of $\log _{10}\left(L_{\mathrm{X}} / \mathrm{erg} \mathrm{s}^{-1}\right)=30.37$, corresponding to a fractional X-ray luminosity of $\log _{10}\left(L_{\mathrm{X}} / L_{\mathrm{bol}}\right)=-3.22$. The X-ray luminosity of V1298 Tau is essentially consistent with that of a saturated X-ray emitter (Wright et al. 2011) and similar to that for other pre-main-sequence fast-rotating stars. The predicted X-ray flux may also be calculated from the Rossby number, $R o=P_{\text {rot }} / \tau_{c}$, where $\tau_{c}$ is the convective turnover time. For V1298 Tau, $R o=0.173$, where $\tau_{c}=16.518$ days was calculated from the $\left(V-K_{s}\right)_{0}$ color (Wright et al. 2011). From empirical relations (Wright et al. 2011), which suggest V1298 Tau is just below the saturated regime, the predicted fractional X-ray luminosity is $\log _{10}\left(L_{\mathrm{X}} / L_{\mathrm{bol}}\right)=-3.47$. Using both the measured and predicted $\log _{10}\left(L_{\mathrm{X}} / L_{\mathrm{bol}}\right)$ values, the age of V1298 Tau implied by an empirical X-ray-age relation (Mamajek \& Hillenbrand 2008) is 12-24 Myr, consistent with the age found in a Hertzpsrung-Russell diagram. 

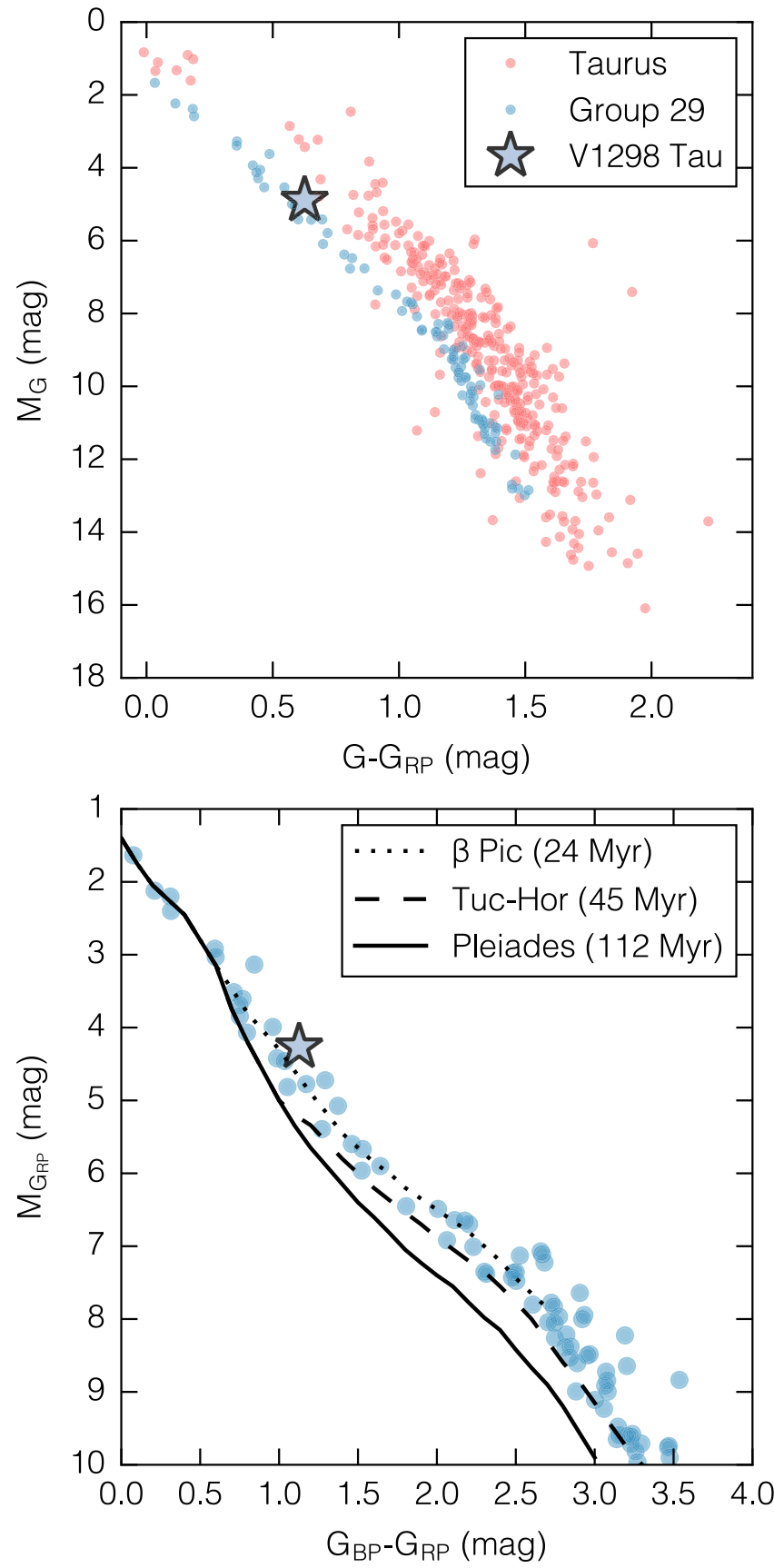

Figure 5. Top: V1298 Tau (blue star) compared to Taurus-Auriga members (red circles) and proposed members of Group 29 (blue circles) in a colorabsolute magnitude diagram. Bottom: V1298 Tau in comparison to empirical fits to the single star sequences of two moving groups and one open cluster (Luhman 2018).

\subsection{Transit Model Fitting}

We fit Mandel \& Agol (2002) analytic transit models to the $K 2$ photometry using a combination of the PYTRANSIT package (Parviainen 2015) and EMCEE, a Python implementation of the affine invariant Markov chain Monte Carlo (MCMC) ensemble sampler (Goodman \& Weare 2010; Foreman-Mackey et al. 2013). The transit model parameters sampled were the orbital period $(P)$, the time of mid-transit $\left(T_{0}\right)$, the planet-to-star radius ratio $\left(R_{\mathrm{P}} / R_{*}\right)$, the scaled semimajor axis $\left(a / R_{*}\right)$, and the cosine of the inclination $(\cos i)$, and two parameter combinations of the eccentricity and longitude of periastron, $(\sqrt{e} \cos \omega, \sqrt{e} \sin \omega)$.
We assumed a quadratic limb-darkening law, imposing Gaussian priors on the linear and quadratic coefficients. The centers and widths of the limb darkening coefficient priors $\left(u_{1}=0.621 \pm 0.023, u_{2}=0.103 \pm 0.015\right)$ were determined from tabulated values appropriate for the temperature and surface gravity of V1298 Tau (Claret et al. 2012, 2013). Using an approximate formula for the mean stellar density in the case of eccentric orbits (Kipping 2010), we additionally applied a Gaussian prior on the light curve-derived stellar density (which is a function of period, $a / R_{\star}, e$, and $\omega$ ) with a center and width of $0.697 \mathrm{~g} \mathrm{~cm}^{-3}$ and $0.225 \mathrm{~g} \mathrm{~cm}^{-3}$, respectively. Model transit profiles were numerically integrated to match the $1766 \mathrm{~s}$ cadence of the $K 2$ observations. The target probability density sampled was therefore

$$
\begin{aligned}
\ln \mathcal{L}= & -\frac{1}{2} \chi^{2}-\frac{1}{2} \frac{\left(\rho_{\star}-\mu_{\rho_{\star}}\right)^{2}}{\sigma_{\rho_{\star}}^{2}} \\
& -\frac{1}{2} \frac{\left(u_{1}-\mu_{u_{1}}\right)^{2}}{\sigma_{u_{1}}^{2}}-\frac{1}{2} \frac{\left(u_{2}-\mu_{u_{2}}\right)^{2}}{\sigma_{u_{2}}^{2}}
\end{aligned}
$$

where the first term describes the likelihood and the last three terms describe the priors on the mean stellar density and limbdarkening parameters.

Convergence was assessed iteratively until the following criteria were met for each directly sampled parameter: (1) the chain length exceeded 50 times the autocorrelation length, and (2) the autocorrelation length estimate changed by $<2 \%$ from the prior iteration. We discarded the first $10 \times\left\langle\tau_{\text {acor }}\right\rangle$ steps as burn-in, where $\left\langle\tau_{\text {acor }}\right\rangle$ is the average autocorrelation length across all parameters. In addition to the fit described above, we performed a circular orbit fit with no prior directly applied to the mean stellar density. This secondary fit was used in an analysis of the host star's evolutionary stage. The radius ratio inferred from the circular orbit fit is nearly indistinguishable from that found for the eccentric fit, $\left(R_{P} / R_{\star}\right)_{\text {iirc }}=0.0713_{-0.0007}^{+0.0012}$. The results of the transit modeling and derived planet parameters are summarized in Table 3 under the Fit 1 columns.

As mentioned earlier, several in-transit observations are outliers with no easily discernible nature (i.e., these observations are not obviously affected by spacecraft systematics). Most of these outliers serve to diminish the transit depth. To determine the effect of the outlying observations on the inferred planetary radius we performed transit fits that both included and excluded these outliers. We adopt the fit which excludes the putative spot-crossing events because the scatter about the lower envelope of the transit profile is smaller. When including all observations, the inferred planet size is $1 \sigma$ smaller than our adopted value $\left(R_{\mathrm{P}}=0.86 \pm 0.05 R_{\mathrm{Jup}}\right)$. Future observations in the infrared, where the amplitude of stellar variability is smaller, may measure the planetary radius more securely.

An unassociated star to the southwest of V1298 Tau is partially contained within the $K 2$ photometric aperture (Figure 10). We show in Section 3.11 that this star is not responsible for the transits, but we consider here the effect of flux dilution on the inferred planet radius. In the presence of light from another star, the ratio of the true planet radius to the observed planet radius is given by $R_{\mathrm{P}, \text { true }} / R_{\mathrm{P}, \text { obs }}=\sqrt{1+F_{2} / F_{1}}$, where $F_{2} / \mathrm{F}_{1}$ is the optical flux ratio between the two stars (Ciardi et al. 2015). The nearby star is $6.3 \mathrm{mag}$ fainter than V1298 Tau in the Kepler bandpass, and thus impacts the planet 


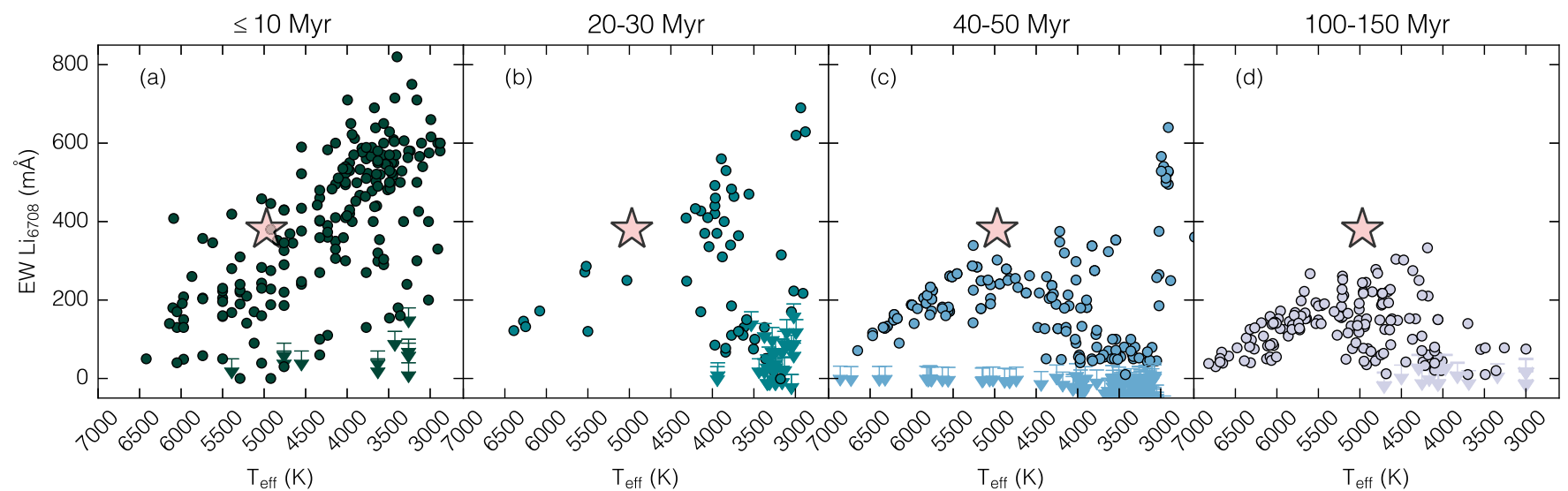

Figure 6. Lithium depletion in young associations; relationship between effective temperature and Li $6708 \AA$ A equivalent width for members of various young associations, moving groups, and clusters. V1298 Tau (pink star) is shown for comparison. (a) Taurus-Auriga, $\eta$ Chamaeleontis, and TW Hydrae. (b) $\beta$ Pictoris moving group and NGC 1960. (c) Tucanae-Horologium, Carina, Columba, IC2602, and IC2391. (d) Pleiades and AB Doradus moving group.
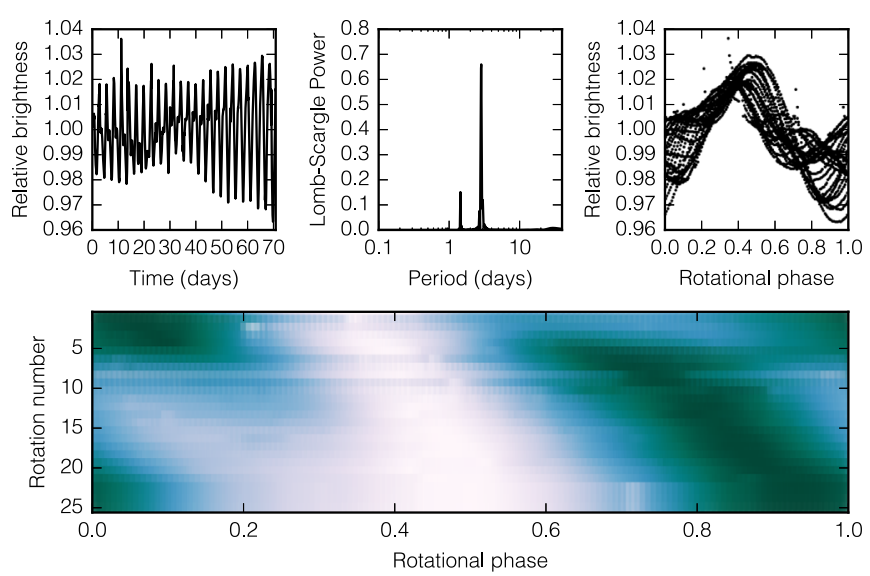

Figure 7. Stellar rotation period. Top: from left to right, the full $K 2$ light curve of V1298 Tau, a Lomb-Scargle periodogram of the $K 2$ time series photometry with peak power at 2.85 days and a secondary peak at the second harmonic, and the $K 2$ photometry phased on the rotation period. Bottom: waterfal diagram visualization of the brightness evolution of V1298 Tau throughout the $K 2$ campaign.

radius by $<0.2 \%$, which is much smaller than the stellar radius uncertainty.

For planets with ingress and egress durations that are sufficiently resolved in time, the eccentricity can be derived from MCMC sampling and a loose stellar density prior (Dawson \& Johnson 2012). From the transit fit, we find the marginalized posterior density in eccentricity places limits of $e<0.16,0.51,0.71$ at $68.3 \%, 95.5 \%$, and $99.7 \%$ confidence, respectively (Figure 11). While transit-derived eccentricities can be robust, we stress that radial velocity monitoring or secondary eclipse timing is required to constrain the eccentricity with higher precision and confidence.

\subsection{Simultaneous Variability and Transit Fits}

To investigate the impact of our variability detrending on the inferred planet parameters, we also performed simultaneous fits to the stellar variability and planet transits using the exoplanet (Foreman-Mackey et al. 2019), starry (Luger et al. 2019), and PyMC3 (Salvatier et al. 2016) packages. We again used the EVEREST 2.0 light curve for this fit. Observations with quality bit flags in the range of $[1,17]$ were discarded.

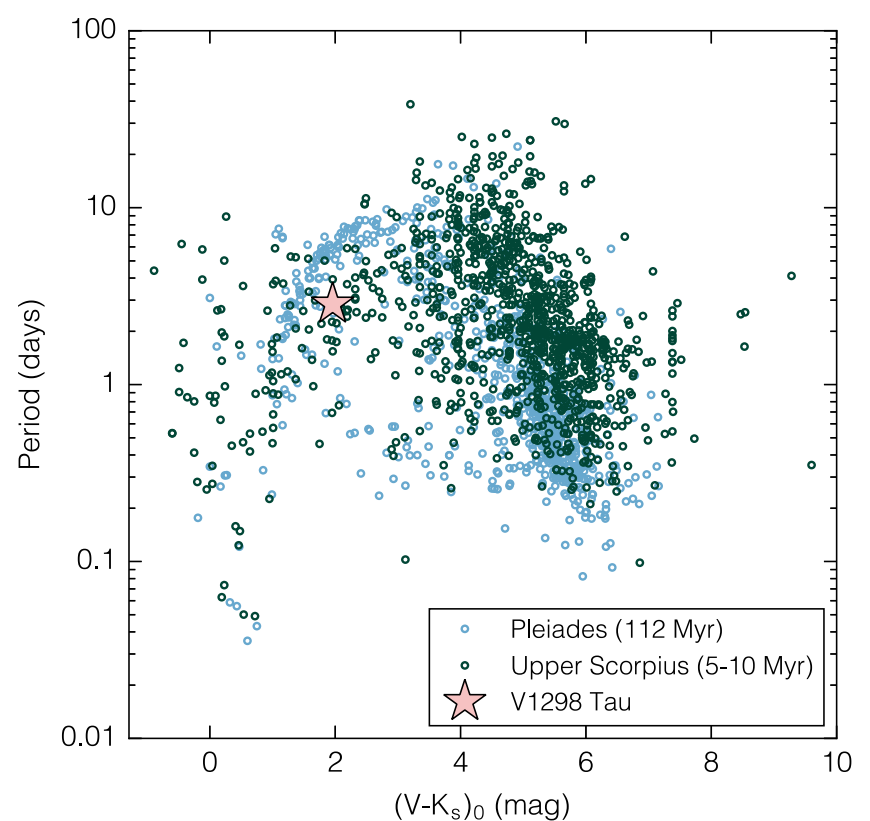

Figure 8. Relationship between colors and variability periods for members of the Upper Scorpius OB association (dark green) and the Pleiades open cluster (light blue), with V1298 Tau (pink star) shown for comparison. Note that the shortest periods at the blue end are due to pulsations rather than surface rotation. Data originate from Rebull et al. $(2016,2018)$.

We constructed a PyMC3 model to describe the light curve using the following parameters: the mean out-of-transit flux $(\langle f\rangle)$, quadratic limb-darkening coefficients $\left(u_{1}, u_{2}\right)$, stellar mass $\left(M_{\star}\right)$ and radius $\left(R_{\star}\right), \log$ of the orbital period $(\ln P)$, time of mid-transit $\left(T_{0}\right)$, radius ratio $\left(R_{\mathrm{P}} / R_{\star}\right)$, impact parameter $(b)$, eccentricity $(e)$, longitude of periastron $(\omega)$, and several hyperparameters to describe the stellar variability using a GP.

We used the "rotation term" GP kernel in exoplanet, which models rotationally modulated variability as a mixture of two stochastically driven, damped simple harmonic oscillators with undamped periods of $P_{\text {rot }}$ and $P_{\text {rot }} / 2{ }^{16}$ The hyperparameters sampled for the GP were the log of the variability

\footnotetext{
16 https://exoplanet.dfm.io/en/stable/user/api/\#exoplanet.gp.terms. RotationTerm
} 


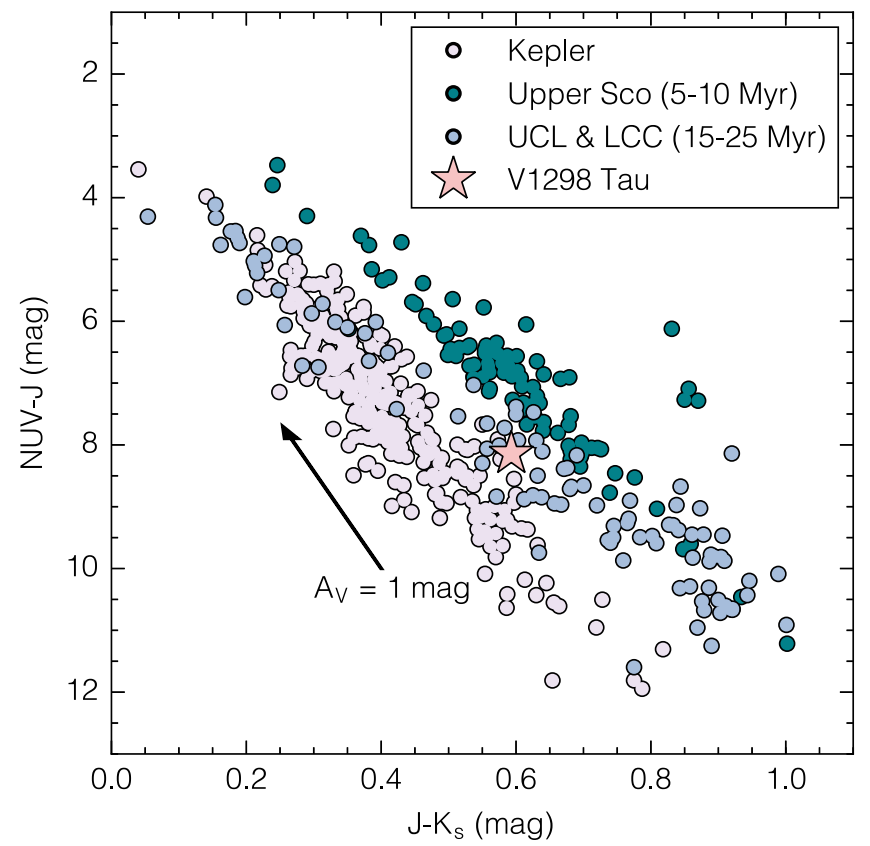

Figure 9. Near-ultraviolet and infrared color-color diagram. V1298 Tau exhibits excess ultraviolet emission, consistent with similarly young (15-25 Myr) stars in Upper Centaurus-Lupus (UCL) and Lower CentaurusCrux (LCC), but at a lower level than stars in Upper Scorpius (5-10 Myr). The Kepler sample is shown for comparison. The photometry has not been corrected for extinction. A reddening vector is shown for reference. Photometry originates from 2MASS (Skrutskie et al. 2006) and GALEX (Martin et al. 2005)

amplitude $(\ln A)$, the $\log$ of the primary variability period ( $\ln P_{\text {rot }}$ ), the $\log$ of the quality factor $-1 / 2$ for the secondary oscillation $\left(\ln Q_{0}\right)$, the $\log$ of the difference between the quality factors of the first and second modes $(\ln \Delta Q)$, and the fractional amplitude of the secondary mode relative to the primary mode (a mixture term referred to here as "mix"). In addition to the hyperparameters described above, a "jitter" parameter $\left(\ln S_{2}\right)$ was introduced to account for excess white noise.

For uninformative sampling of the quadratic limb-darkening coefficients, exoplanet uses the parameterization recommended by Kipping (2013a). Additionally, for efficient sampling of the radius ratio and impact parameter, exoplanet uses the joint parameterization of those parameters suggested by Espinoza (2018). For this fit, a $\beta$ distribution prior was assumed for the eccentricity, with the assumed values $a=0.867, b=3.03$ as recommended by Kipping (2013b).

We masked observations within $12 \mathrm{hr}$ of each transit in order to ensure that the transits did not influence the variability model and performed an initial optimization of the PyMC3 model. After the initial optimization, $7 \sigma$ outliers were masked (where $\sigma$ was defined as the rms of the residuals of this initial fit). This step effectively masked both in-transit and out-of-transit outliers from the sampling procedure that followed. A new optimization was performed, followed by MCMC sampling with the no U-turns step method (Hoffman \& Gelman 2014). We ran four chains with 500 tuning steps to learn the step size, 4500 tuning iterations (tuning samples were discarded), a target acceptance of $99 \%$, and 3000 draws for a final chain length of 12,000 in each parameter. Convergence was assessed using the Gelman-Rubin diagnostic (Gelman \& Rubin 1992), which was below 1.001 for each parameter.
The procedures described above were adapted from exoplanet tutorials published online. ${ }^{17}$ Results from the simultaneous variability and transit fits, along with the priors imposed on all of the sampled parameters, are summarized in Table 3 under the Fit 2 columns. Figure 12 shows the GP model around the transits, and Figure 13 shows the phasefolded transit and residuals from the simultaneous variability and transit fit.

\subsection{Centroid Motion Analysis}

Evidence of offsets in the point-spread function (PSF) centroid during transit are indicative of a transit occurring due to a contaminant or background star with a transient nature (e.g., a background eclipsing binary, Thompson et al. 2018). Based on a simple centroiding test, accounting for the $K 2$ roll motion, the PSF centroids during the expected transit of V1298 Tau $\mathrm{b}$ are consistent at the $\lesssim 1 \sigma$ level with the out-of-transit centroids (Figure 14). This suggests that the transit signal is not due to a background eclipsing binary and is consistent with originating from the target star.

\subsection{Limits on Companions from the Transit Shape}

The transit shape constrains the probability of hierarchical triple scenarios in which the observed transit signal is due to an eclipsing binary or transiting planet host which is distinct from, but gravitationally bound to, V1298 Tau. In such scenarios, the flux dilution from V1298 Tau itself requires larger radius ratios (and thus more V-shaped eclipses) between the eclipsing companions in order to reproduce the observed transit depth. To estimate the relative likelihood of these scenarios, we performed Levenberg-Marquardt least-squares fits (with free parameters $R_{\mathrm{P}} / R_{*}, a / R_{*}, \cos i$ ) to the transit profile over a grid of assumed optical contrasts between V1298 Tau and the putative eclipsing companions.

In this case the model time series is given by the equation

$$
f_{\text {dil }}(t)=\frac{f(t)+F_{1} / F_{2}}{1+F_{1} / F_{2}},
$$

where $f(t)$ is the normalized eclipse time series in the absence of dilution and $F_{1} / F_{2}$ is the flux ratio between the primary star and the eclipsing companions.

For each contrast value and best-fit eclipse profile, we recorded the $\chi^{2}$ value. Using the Akaike Information Criterion we then evaluated the relative likelihoods of models which assume dilution from the primary star. We found that a model with a companion contrast of $\Delta \mathrm{mag}=1$ is $5.6 \times 10^{-4}$ times as likely as one with $\Delta \mathrm{mag}=0$. By comparison, a model assuming dilution from an equal-brightness companion is found to be 0.057 times as likely as the best-fit model assuming no additional companion. We also performed an MCMC fit for a diluted transit model, sampling the parameters $R_{\mathrm{P}} / R_{*}, a / R_{*}$, $\cos i$, and $\Delta \mathrm{mag}$ and using the same convergence criteria utilized in the transit fit described earlier. This analysis leads to a constraint of $\Delta \mathrm{mag}<0.76$ for a putative companion at 99.7\% confidence. Although the statistical tests described above suggest a stringent limit on the contrast of a putative companion, we adopt a more conservative limit of $\Delta$ mag $<4$, based on the shapes of the best-fit transit profiles after accounting for dilution (Figure 15), for plausible hiearchical

\footnotetext{
17 https://exoplanet.dfm.io/en/stable/
} 
Table 3

V1298 Tau Light Curve Modeling Results

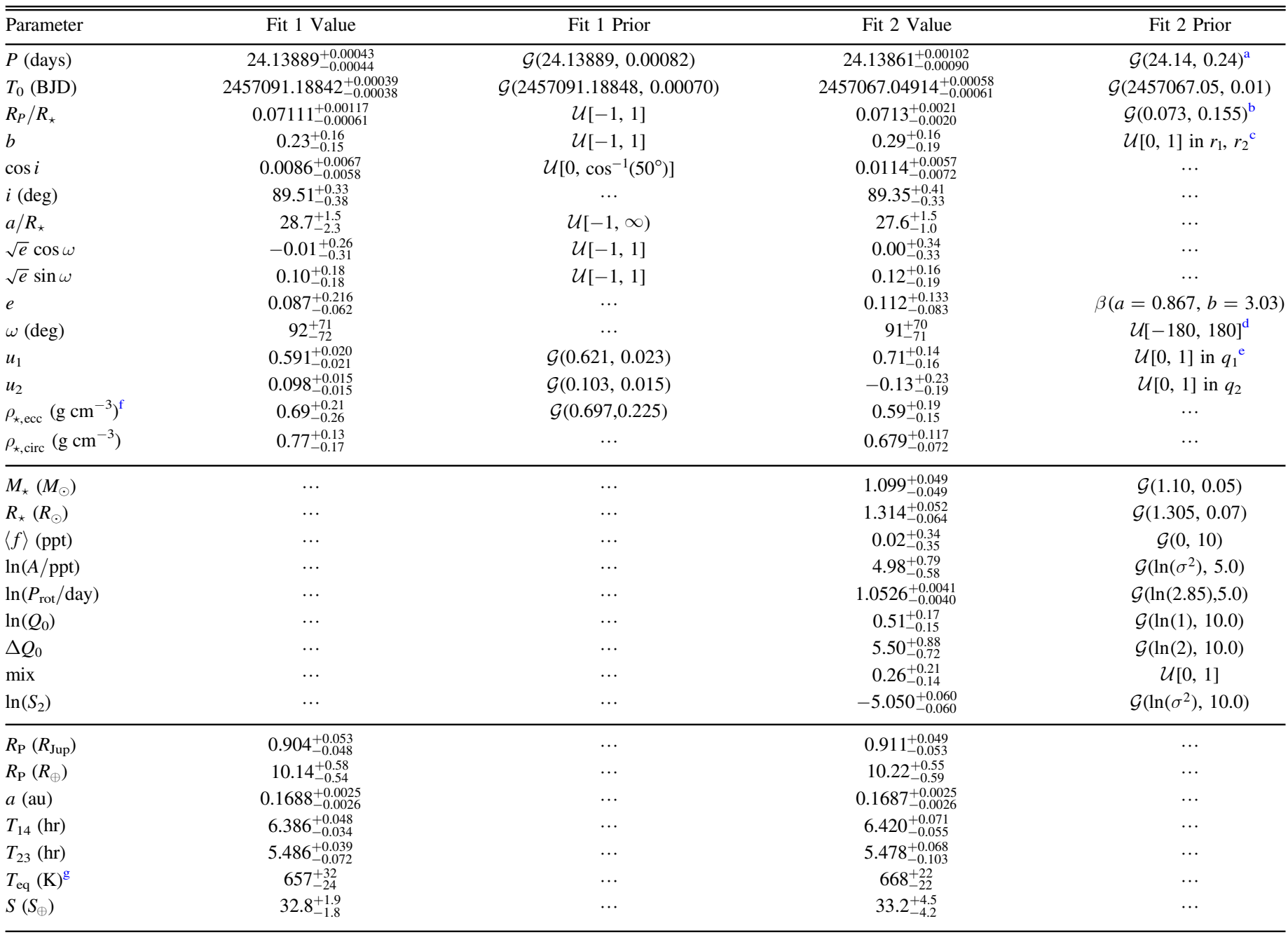

Notes. Priors are noted for those parameters which were directly sampled. $\mathcal{G}$ : Gaussian; $\beta$ : beta distribution; $\mathcal{U}$ : uniform; $\sigma$ : standard deviation in flux (191 ppt). Quoted transit parameters and uncertainties are medians and 15.87\%,84.13\% percentiles of the posterior distributions. We recommend adopting the values from Fit 2.

${ }^{a}$ Sampling performed in $\ln (P)$.

${ }^{\mathrm{b}}$ Sampling performed in $\ln \left(R_{P} / R_{\odot}\right)$.

${ }^{c}$ Joint sampling of impact parameter and radius ratio performed using Espinoza (2018) parameterization.

${ }^{\mathrm{d}}$ Sampling performed in $(\cos \omega, \sin \omega)$.

${ }^{\mathrm{e}}$ Uninformative sampling of quadratic limb-darkening coefficients performed using parameterization of Kipping (2013a).

${ }^{\mathrm{f}}$ Calculated from Equation (39) of Kipping (2010).

${ }^{\mathrm{g}}$ Calculated assuming an albedo of 0 .

triple scenarios. Using Equation (7) from Ciardi et al. (2015), which assumes the planet transits the primary star, we calculated the true planet radius would be larger by $41 \%$, $22 \%$, and $1 \%$ for putative companions with $\Delta \mathrm{mag}=0,0.76$, and 4 , respectively.

\subsection{Limits on Companions from Imaging}

Daemgen et al. (2015) observed V1298 Tau with AO and the NIRI instrument at Gemini North Observatory on 2011 October 22 11:00 UTC. Those data rule out nearly all scenarios in which V1298 Tau hosts a stellar mass companion with a projected separation of 10.85-1085 au, where we have interpolated between a $20 \mathrm{Myr}$ isochrone to convert nearinfrared contrasts to mass limits (Baraffe et al. 2015). In the time between those observations and our NIRC2 imaging, V1298 Tau moved by 0 "! $120 \pm 0$ ".062 on the sky due to its proper motion. Combining the two epochs of imaging constraints we rule out a vast swath of parameter space involving a background or foreground eclipsing binary that would have been aligned by chance with V1298 Tau during the $K 2$ observations (Figure 16).

We used a galactic structure model (Girardi et al. 2012) to simulate a $1 \mathrm{deg}^{2}$ field centered on V1298 Tau in order to estimate the number of foreground or background stars in the region of parameter space not excluded by our observations. We found that 0.004 sources with $3.4<\Delta V<5.8$ mag are expected in the surrounding 0 !" $1 \times 0$ "! 1 region. An even smaller number of sources are expected to be eclipsing binaries. 

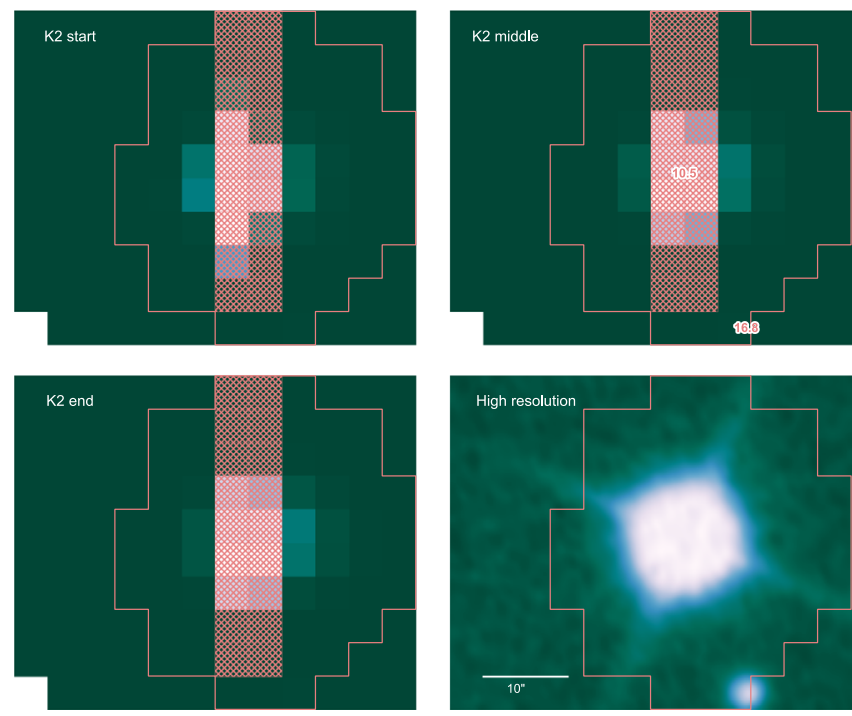

Figure 10. $K 2$ photometric aperture for V1298 Tau. $K 2$ target pixel files for V1298 Tau at the start (top left), middle (top right), and end (bottom left) of Campaign 4. Bottom right: high-resolution image from the Palomar Observatory Sky Survey showing a faint background source to the southwest. The photometric aperture boundary is indicated in pink, with saturated columns shown as hatched regions.

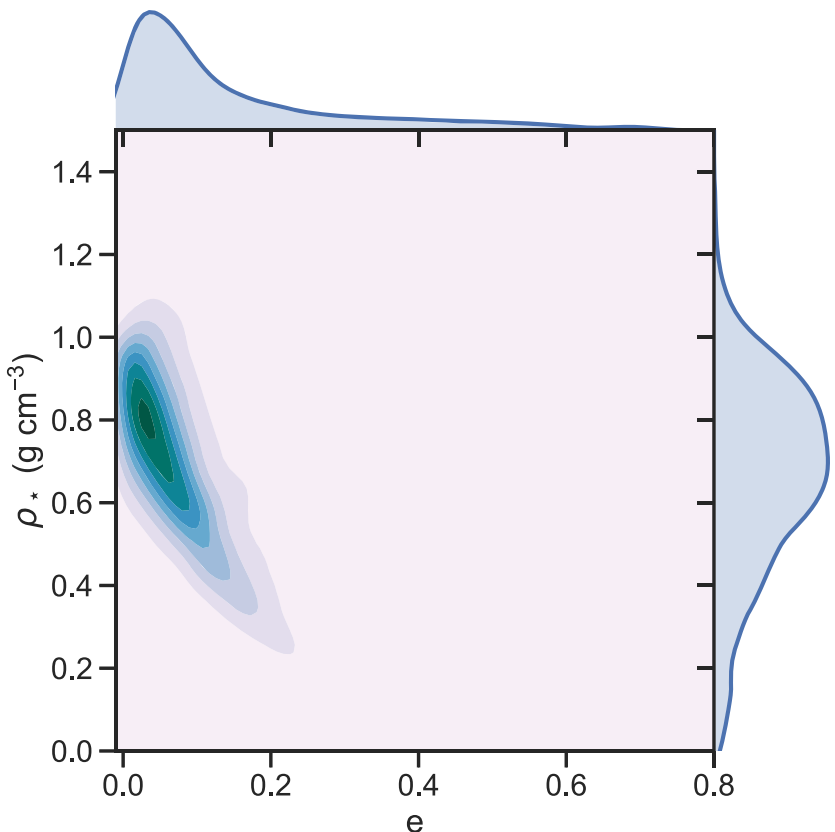

Figure 11. Constraints on eccentricity of V1298 Tau b from the transit fit (Fit 1 , as described in Section 3.4). Shaded contours show the joint posterior probability densities for the eccentricity of V1298 Tau b and mean stellar density from the transit fit. Marginalized probability densities are shown at top and at right.

\subsection{Limits on Companions from Spectroscopy}

We used a $24 \mathrm{yr}$ radial velocity time series (Wichmann et al. 2000; Nguyen et al. 2012; this work), including our newly acquired data, to search for bound companions to V1298 Tau. We assumed an uncertainty of $1 \mathrm{~km} \mathrm{~s}^{-1}$ for each observed radial velocity to allow for the possibility of zero-point offsets between different instruments or other systematic biases. At each point in a grid of orbital period and companion mass we simulated 1000 circular spectroscopic binary orbits, sampled at

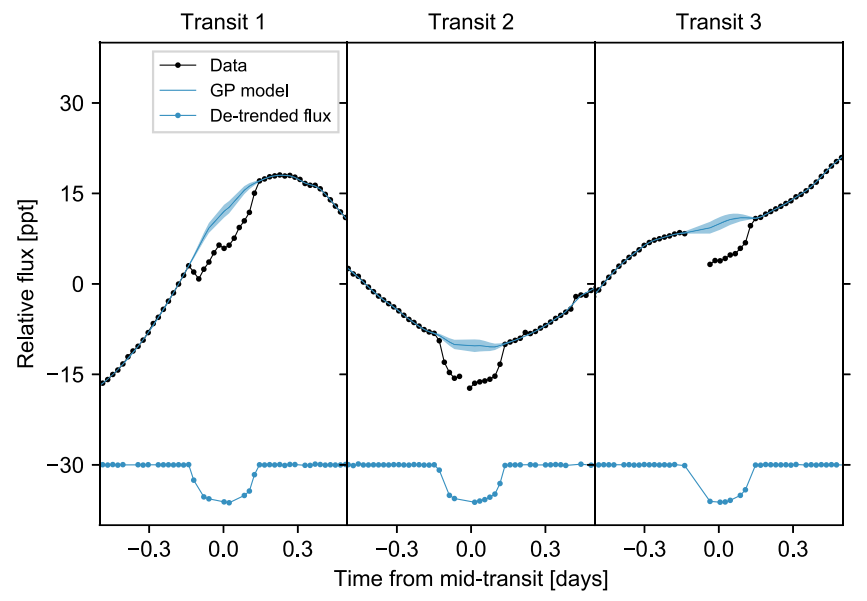

Figure 12. Transits of V1298 Tau b in the EVEREST 2.0 light curve (black dotted line) with the GP model from the simultaneous variability and transit fits described in Section 3.5 shown by the blue line. The shaded bands show the $3 \sigma$ error contours of the GP model. The de-trended flux (data-median GP modeloffset) is shown by the blue dotted line below each transit.

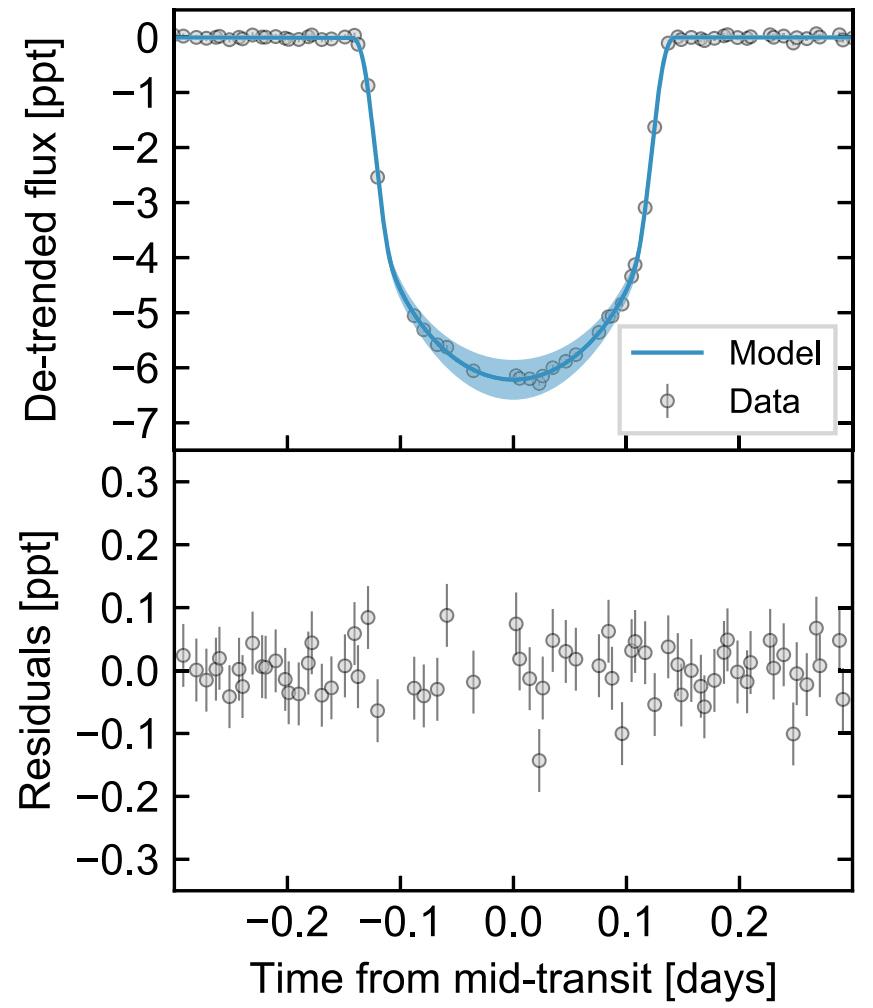

Figure 13. Top: phase-folded transit observations (points) and median model (solid line) from the simultaneous variability and transit fits described in Section 3.5 (Fit 2). The $1 \sigma$ error contours are shown by the shaded bands. Bottom: residuals from the median model.

the times of the observations. For each simulated orbit, the inclination was drawn randomly from a uniform distribution in $\cos i$ and the phase was drawn randomly from a uniform distribution between $[0,2 \pi]$. To simulate radial velocity jitter, we added Gaussian noise with an amplitude of $200 \mathrm{~m} \mathrm{~s}^{-1}$ to each model. At each point in the period-companion mass plane we then determined the detection probability as the fraction of successfully detected simulated orbits. An individual simulated orbit was considered successfully detected if the $\Delta \chi^{2}$ value between the simulated radial velocities and the null hypothesis 


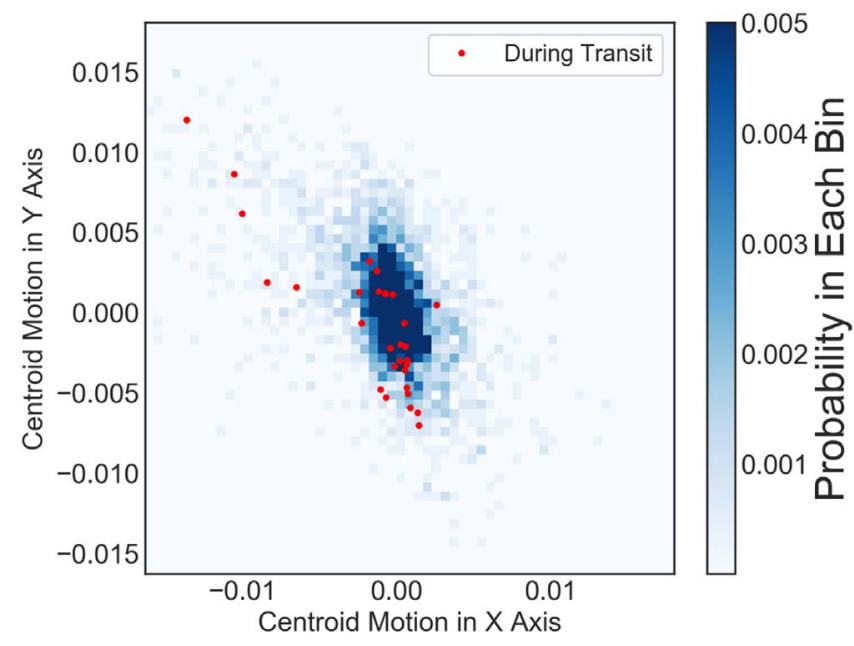

Figure 14. Centroid motion of V1298 Tau; motion of the point-spread function centroid of V1298 Tau throughout the K2 campaign (two-dimensional histogram). The centroid motions during the transits of V1298 Tau b are shown as red points, and are consistent with with the distribution of out-oftransit centroid shifts at the $\lesssim 1 \sigma$ level. The five clear outliers correspond to observations which occurred immediately after a loss of fine pointing by the telescope.
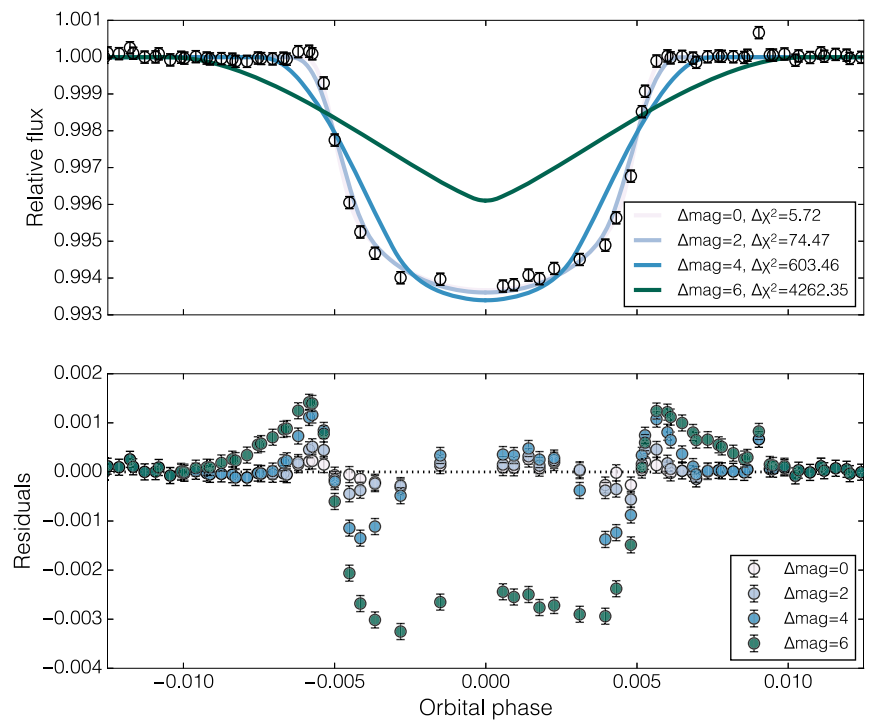

Figure 15. Constraints on companions to V1298 Tau from the transit shape; best-fit transit models after accounting for dilution from V1298 Tau. For large optical contrasts between V1298 Tau and a putative companion, models cannot reproduce the observed transit shape and depth. For each assumed contrast, the $\Delta \chi^{2}$ value between the hierarchical triple model and the single star model is indicated. Scenarios involving a companion with $\Delta \mathrm{mag}>4$ are ruled out.

of constant velocity exceeded 20. From this analysis we are able to rule out a wide range of brown dwarf and stellar companions with orbital periods between 1 and 10,000 days (Figure 17).

In a search for secondary spectral lines in the HIRES spectrum (Kolbl et al. 2015) we found no stars brighter than $5 \%$ of the brightness of the primary and with a projected separation of $\leqslant 0$ ". 4 . We note that this search is only sensitive to stars with radial velocity separations $>50 \mathrm{~km} \mathrm{~s}^{-1}$ (corresponding approximately to two linewidths of V1298 Tau), as the two sets of spectral lines would otherwise be indistinguishable.

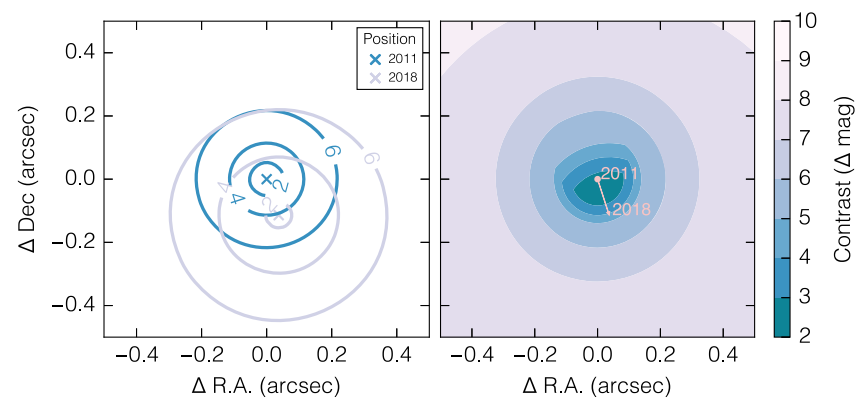

Figure 16. Multi-epoch adaptive optics imaging of V1298 Tau. Left: radial contrast contours in the $1^{\prime \prime}$ region surrounding V1298 Tau from multi-epoch adaptive optics imaging. Right: combination of the individual constraints results in a minimum contrast of $\Delta K=2.1 \mathrm{mag}$ everywhere in the surrounding region, including behind the position of V1298 Tau during the $K 2$ observations.

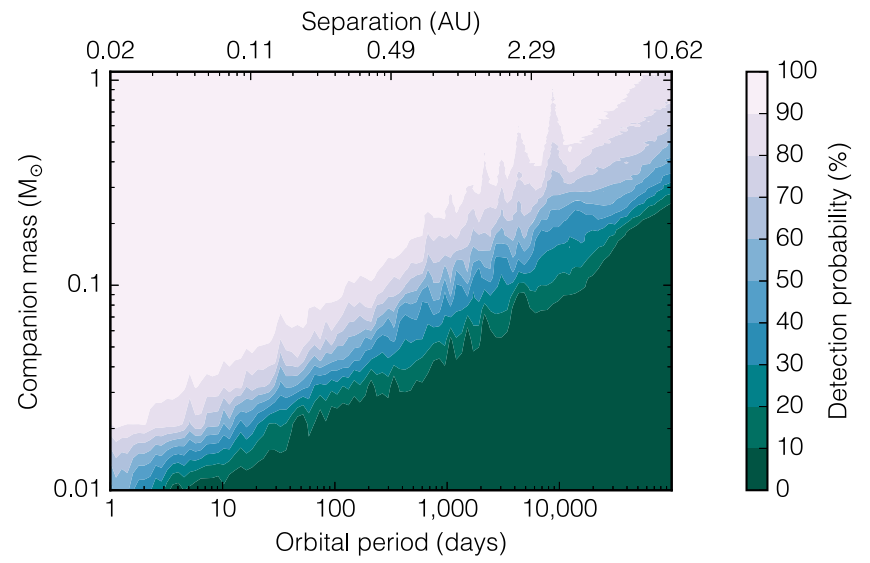

Figure 17. Radial velocity limits on companions to V1298 Tau; detection probability in the period-companion mass plane for simulated spectroscopic binaries given the $24 \mathrm{yr}$ radial velocity time series. The axis at the top indicates the physical separation for a $0.5 M_{\odot}$ companion. The $95 \%$ detection probability contour is depicted in Figure 18.

\subsection{Limits on Companions from Astrometry}

Gaia resolves double stars, either associated or unassociated, outside of $1^{\prime \prime}$ down to optical contrasts of $6 \mathrm{mag}$ and irrespective of a companion's position angle (Ziegler et al. 2018). Only one other star was detected by Gaia within the boundaries of the $K 2$ photometric aperture. We show later that star is too faint to be a false positive. At smaller separations, the goodness-of-fit of the Gaia astrometric solution provides another means of assessing multiplicity. A previous study of exoplanet host stars with closely projected companions showed that the Gaia astrometric fit metrics can be used to reliably detect companions with separations of $0 . " 08-1^{\prime \prime}$ and optical contrasts $<2$ mag (Rizzuto et al. 2018). Furthermore, exoplanet host stars with detected companions at projected separations of $0 . " 05-1^{\prime \prime}$ typically have astrometric goodness-of-fit values $>20$ with excess astrometric noise often detected at $5 \sigma$ significance (Evans 2018). In the case of V1298 Tau, the goodness-of-fit in the along-scan direction is 9.0, with zero detectable excess astrometric noise. While not conclusive, the Gaia data suggest V1298 Tau is unlikely to host a stellar companion with a mass above $0.5 M_{\odot}$ (corresponding to a model-derived optical contrast of 2 mag at $20 \mathrm{Myr}$; Baraffe et al. 2015) and separation greater than 0 ". $08(8.7 \mathrm{au})$. 

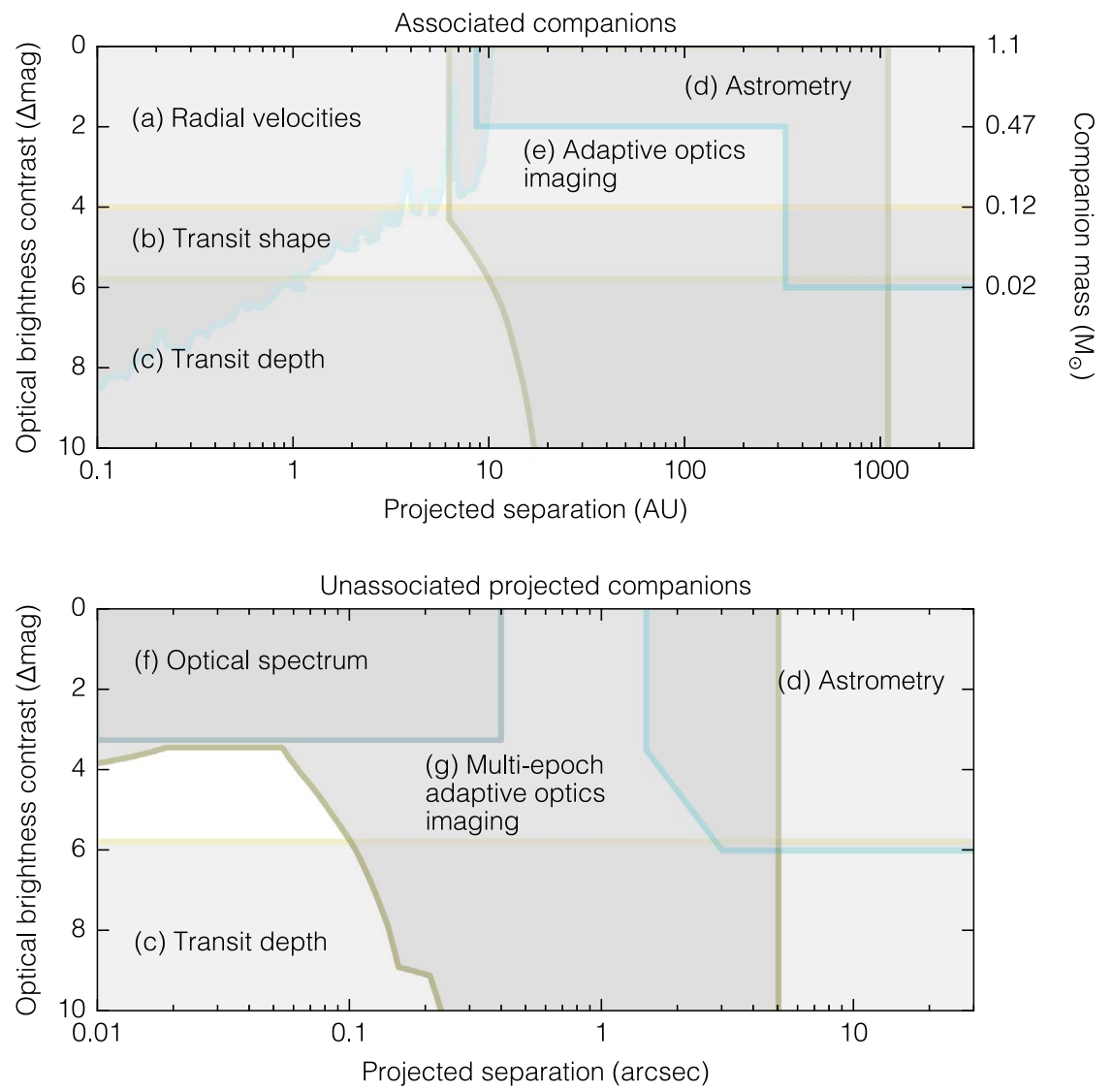

Figure 18. Constraints on astrophysical false-positive scenarios. Regions of the separation-contrast plane excluded by observations are shown for associated companions at the top, and unassociated companions below. (a) Sustained radial velocity monitoring rules out associated eclipsing binaries at close separations. (b) The transit shape rules out certain hierarchical triple scenarios at any separation. (c) The observed transit depth sets an upper limit of 5.7 mag to the optical contrast between V1298 Tau and a putative false positive, whether it be associated or not. (d) Gaia astrometry resolves point sources beyond 1", while the astrometric goodness-of-fit is a predictor of associated companions outside of 80 mas. (e) Adaptive optics imaging rules out wide associated companions. (f) A search for secondary spectral lines would have detected bright eclipsing binaries at close separations as long as the velocity separation with V1298 Tau was $>50 \mathrm{~km} \mathrm{~s}^{-1}$. (g) Multi-epoch adaptive optics combined with the proper motion of V1298 Tau eliminates the bulk of unassociated false-positive scenarios. The probability of an unassociated eclipsing binary residing within 0 ! 1 , where our observations are not sensitive, is $<4 / 1000$.

\subsection{False-positive Scenario Assessment}

False-positive signals in transit surveys can be due to an eclipsing binary or a planet transiting a star other than the assumed host. We consider each false-positive scenario in detail below (summarized in Figure 18), and conclude that the interpretation of a Jupiter-sized planet transiting V1298 Tau is the one most consistent with observations.

An eclipsing binary can dim by a maximum of $100 \%$, which sets a firm upper limit of $5.7 \mathrm{mag}$ to the optical contrast between V1298 Tau and a putative false positive capable of reproducing the observed transit depth. Although an unassociated star is included in the target pixel files for V1298 Tau, it is too faint to reproduce the observed transit depth and we recovered the transits from a small photometric aperture excluding that star. Inside of 0 ." 1 , assuming an unassociated eclipsing binary would not be co-moving with V1298 Tau, we effectively ruled out background or foreground stars by leveraging the proper motion of V1298 Tau between two epochs of AO imaging separated by $6 \mathrm{yr}$. At separations of 0 "' $1-20^{\prime \prime}$, AO imaging, all-sky photometric surveys, and Gaia rule out additional stars bright enough to be a false positive. Furthermore, a time-series analysis of the K2 PSF indicates that the centroid shifts during transits are consistent with those signals originating from V1298 Tau.
False-positive scenarios involving hierarchical triples, i.e., an eclipsing binary or a transiting planet host which is gravitationally bound to V1298 Tau, are similarly disfavored. At close separations $(<10 \mathrm{au})$, we used a $24 \mathrm{yr}$ radial velocity time series (Wichmann et al. 2000; Nguyen et al. 2012) to rule out stellar and brown dwarf companions in a mass-dependent manner, allowing for isotropically distributed inclinations of a putative companion. At intermediate separations ( 10-1000 au), previously published (Daemgen et al. 2015) and newly acquired AO imaging rules out nearly all stellar companions. Furthermore, the lack of astrometric noise detected with Gaia suggests there are no companions down to $\sim 0.5 M_{\odot}$ in the $\sim 10-300$ au range. At projected separations beyond $1000 \mathrm{au}$, stellar mass and some brown dwarf companions would have been detected by Gaia or all-sky surveys. Further discussion of false-positive scenarios is presented in Appendix A.

\subsection{Limit on the Planet Mass}

Using the RADVEL radial velocity orbit-fitting code (Fulton et al. 2018), we fit the PRV measurements of V1298 Tau in order to find an upper limit to the planet's mass (Figure 19). Due to the sparse orbital phase coverage of our data and to the high radial velocity jitter of the star, this upper limit is very likely to be conservative. We assumed a circular orbit for our 

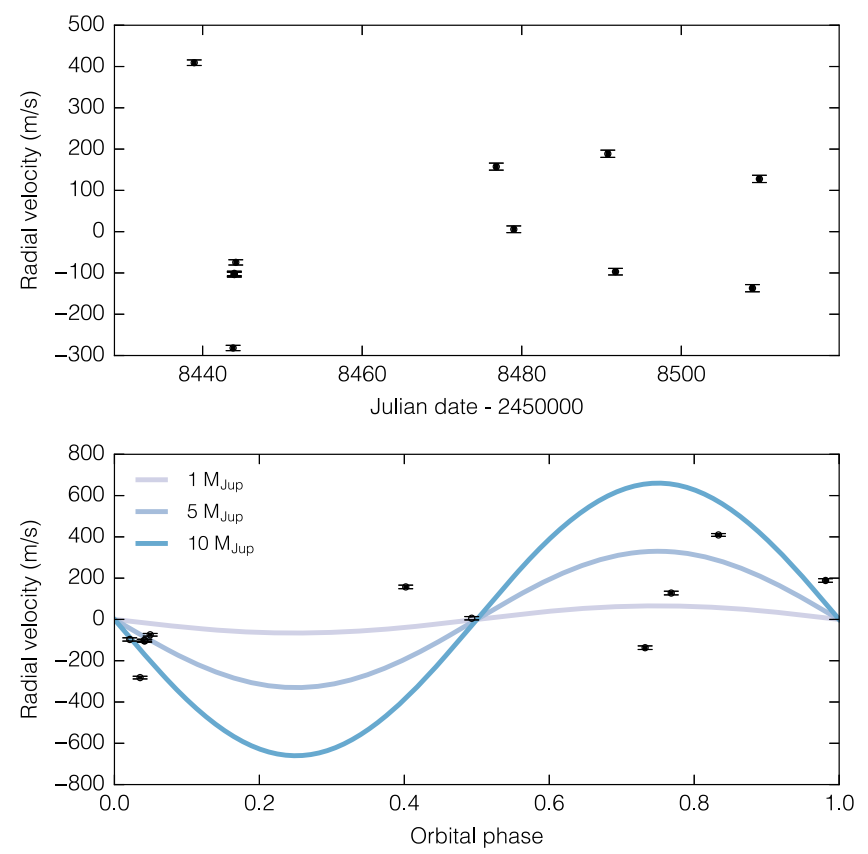

Figure 19. Precision radial velocities of V1298 Tau. Top: Keck/HIRES precision radial velocity time series of V1298 Tau. Bottom: PRV measurements as a function of orbital phase, with representative circular Keplerian orbits shown for reference.

fits, imposing Gaussian priors on the period and time of inferior conjunction corresponding to the values found from the transit fit (Table 2). Furthermore, the jitter amplitude was fixed to $200 \mathrm{~m} \mathrm{~s}^{-1}$, corresponding approximately to the rms dispersion between all measurements. The parameter space defined by the period, time of inferior conjunction, radial velocity semiamplitude, and velocity zero-point was then explored through MCMC sampling. The resulting posterior of the radial velocity semi-amplitude provides a $3 \sigma$ upper limit to the planet mass of $M_{\mathrm{P}}<8.3 M_{\text {Jup }}$.

\section{Discussion}

Without a measurement of the planet's mass, it is difficult to place V1298 Tau b in proper context with respect to the observed exoplanet population. A priori, the most likely hypothesis from an occurrence rate standpoint is that V1298 Tau b has a mass more similar to typical Kepler planets, with a radius that has not yet contracted due to its young age. Of the confirmed transiting exoplanets with measured masses and orbital periods in the 10-100 day range, the median mass is $11 M_{\oplus}$ with a 16 th-84th percentile range of $4-58 \mathrm{M}_{\oplus}$. Depending on the exact core mass and the mass and initial entropy of the $\mathrm{H} / \mathrm{He}$ envelope, evolution models of low-mass planets $\left(<100 M_{\oplus}\right)$ do indeed predict large radii $\left(\sim 10 R_{\oplus}\right)$ at ages of 20-30 Myr (Lopez \& Fortney 2013; Owen \& Wu 2013; Jin et al. 2014; Chen \& Rogers 2016). Interestingly, at a separation of $\approx 0.17 \mathrm{au}, \mathrm{V} 1298$ Tau b should be largely unaffected by photoevaporation (Owen \& Wu 2013). Thus, if V1298 Tau b does indeed have a low mass more representative of the Kepler population, it may be a particularly valuable benchmark for planet evolution models; V1298 Tau b would provide an opportunity to learn more about the initial conditions of this common class of planet in the absence of substantial photoevaporation.
On the other hand, given that the current limit on the planet's mass is not very restrictive, we must consider the possibility that V1298 Tau b belongs to the class of planets known as "warm Jupiters." The size of V1298 Tau b is significantly smaller than the predictions of many evolutionary models for Jovian-mass planets (Burrows et al. 1997; Baraffe et al. 2003; Fortney et al. 2007; Mordasini et al. 2012; Owen \& Wu 2013), which predict radii of $\gtrsim 1.3 R_{\text {Jup }}$ at an age of $\sim 20 \mathrm{Myr}$. Such a large radius would be possible if V1298 Tau hosts a nearly equal brightness companion, though such a companion should have been detected from the suite of follow-up observations. In fact, the models of Fortney \& Nettelmann (2010) suggest that the combination of age, radius, and separation for V1298 Tau b is inconsistent with a gas-dominated composition for a planet with mass $>0.1 M_{\text {Jup }}\left(32 M_{\oplus}\right)$. However, the observed properties of V1298 Tau b might be reproduced by a planet with a core-dominated composition and mass in the range $0.1-0.3 M_{\text {Jup }}$ (with a core mass of $25 M_{\oplus}$ ). If the true mass of V1298 Tau b is $\gtrsim 0.3 M_{\text {Jup }}$, it may still be considered a warm Jupiter by conventional definitions.

Relative to hot Jupiters, less is known about warm Jupiters, though similar mechanisms are invoked to explain their origins. There is evidence for two distinct populations of warm Jupiters. The majority of these planets are characterized by low eccentricities $(e \lesssim 0.2)$, nearby and coplanar super-Earth companions, and a dearth of external Jovian-mass companions (Dong et al. 2014; Huang et al. 2016). The remaining warm Jupiters are characterized by moderately eccentric $(e \gtrsim 0.4)$ orbits, often accompanied by external Jovian-mass companions which are mutually inclined and apsidally misaligned (Dawson \& Chiang 2014).

In general, warm Jupiters do not have eccentricities large enough to become hot Jupiters through tidal dissipation (Dawson et al. 2015). Furthermore, population synthesis studies examining the outcomes of various high-eccentricity migration scenarios have struggled to reproduce the observed ratio of hot and warm Jupiters (Antonini et al. 2016; Petrovich $\&$ Tremaine 2016). Both of these points of evidence are seen as a major weakness in the high-eccentricity migration hypothesis. However, secular interactions with a neighboring planet could excite the eccentricity of V1298 Tau b at a later stage (Anderson \& Lai 2017).

While the transit fit for V1298 Tau b suggests a low to moderate eccentricity, solutions with $e>0.5$ are not ruled out. However, eccentricities measured from transit photometry (particularly long-cadence Kepler data as in this case) should be regarded with caution. Until the mass and eccentricity of V1298 Tau b are measured through radial velocities or secondary eclipse timing, it remains unclear whether the planet belongs to either of the known warm Jupiter populations. Even if V1298 Tau b is found to have a moderately low eccentricity, long-term radial velocity monitoring is required to test the hypothesis that the planet might have an external companion that is nearby and massive enough to force episodic tidal migration through secular planet-planet interactions. In the absence of such secular interactions, the final semimajor axis can be calculated from the present-day semimajor axis and eccentricity. From the posterior probability densities resulting from the light-curve modeling, we calculated the final semimajor axis, $a_{\mathrm{final}}=a\left(1-e^{2}\right)$, is $>0.13$ au at $95 \%$ confidence, and $>0.09$ au at $99 \%$ confidence. For V1298 Tau 
$\mathrm{b}$ to migrate inwards to $\leqslant 0.1$ au requires an eccentricity of $\geqslant 0.64$.

\section{Conclusions}

We report the detection and validation of a warm Jupitersized planet transiting a young solar analog with an estimated age of 23 Myr. The star and its planet belong to a newly characterized association, named Group 29, in the foreground of the Tau-Aur star-forming region (Oh et al. 2017; Luhman 2018). Through a detailed analysis of the $K 2$ data and follow-on observations, we found no evidence for a stellar companion and we securely ruled out most plausible falsepositive scenarios. The young age of the planetary system challenges, but does not outright exclude, high-eccentricity migration as a viable formation channel. Formation in situ (e.g., Hansen \& Murray 2012; Batygin et al. 2016; Boley et al. 2016) or at a wider separation followed by planet-disk interactions (e.g., Terquem \& Papaloizou 2007; Kley \& Nelson 2012) would be compatible with current observations and not at odds with the star's youth.

The transit profiles exhibit some anomalous features, and it is currently unclear whether the outlying observations are systematic or astrophysical in nature. Transit observations in the infrared, where the amplitude of stellar variability is lower, should yield a more secure measurement of the planet's radius. To measure the planet's mass, it is also preferable to observe in the infrared, as we have measured the optical radial velocity jitter to be $\sim 200 \mathrm{~m} \mathrm{~s}^{-1}$ (compared with the range of predicted Doppler semi-amplitudes of $13-230 \mathrm{~m} \mathrm{~s}^{-1}$ ). We discuss the favorability of V1298 Tau for various follow-up observations further in Appendix B.

Although several groups have published candidate exoplanet catalogs encompassing nearly every $K 2$ campaign, the V1298 Tau system seems to have evaded detection, despite the relatively large transit depth. The most likely explanation seems to be that automated transit search pipelines are not tailored to the high-amplitude, short-timescale variability exhibited by young stars. Customized or more generalized routines that are not susceptible to under- or over-fitting stellar variability are therefore needed to adequately search the light curves of young stars for transiting planets (e.g., Rizzuto et al. 2017).

Currently, there is only one other secure case of an exoplanet transiting a pre-main-sequence star: the Neptune-sized planet K2$33 \mathrm{~b}$ in the 5-10 Myr-old Upper Scorpius OB association (David et al. 2016; Mann et al. 2016b). V1298 Tau b is now the second youngest transiting exoplanet, and the first secure case of a transiting Jovian-sized planet orbiting a pre-main-sequence star. Many of the other transiting planets found in young $(<1 \mathrm{Gyr})$ clusters reside in low-occurrence regions of the period-radius diagram (Figure 20), such as the radius valley or the sub-Jovian desert. V1298 Tau b seems to follow this trend, though it is as yet unclear whether this planet is a Jovian-mass resident of the "period-valley" (Udry et al. 2003; Santerne et al. 2016), a progenitor of the rare sub-Saturn class (Petigura et al. 2017), or perhaps contracting down to the sub-Neptune "main sequence."

From an occurrence rate standpoint, the hypothesis that V1298 Tau b has a mass $<30 M_{\oplus}$ is at least an order of magnitude more likely than the hypothesis that the mass is above this threshold (Howard et al. 2010b; Mayor et al. 2011). Furthermore, at an age of $\sim 23 \mathrm{Myr}$, the planet's size is significantly smaller than expected for Jovian-mass planets

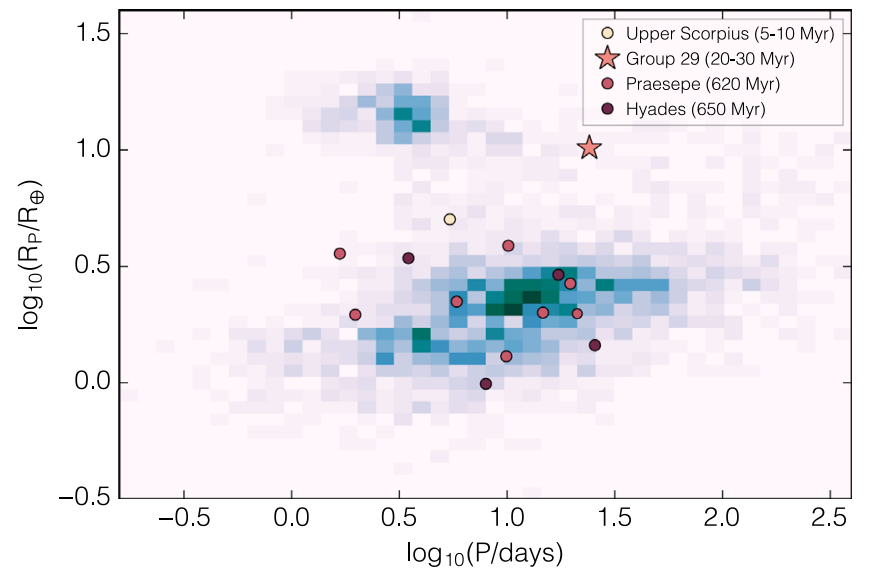

Figure 20. V1298 Tau b (pink star) compared to the sample of confirmed transiting exoplanets (two-dimensional histogram) in the period-radius domain. Transiting planets in young clusters and associations are shown for comparison.

(Burrows et al. 1997; Baraffe et al. 2003; Fortney et al. 2007; Mordasini et al. 2012) but consistent with the radii of low-mass Kepler-type planets for a range of plausible core and envelope masses (Lopez \& Fortney 2013; Owen \& Wu 2013; Jin et al. 2014; Chen \& Rogers 2016).

In general, Kepler planets (i.e., super-Earths and miniNeptunes) are believed to have formed while the gas disk was still present, given that a substantial fraction of them possess an envelope constituting $\sim 1 \%-10 \%$ of the total mass. However, the details of where and when the embryos formed, whether migration plays a significant role, and where the envelopes are accreted are debated (e.g., Terquem \& Papaloizou 2007; Ida \& Lin 2010; Hansen \& Murray 2012; Lee et al. 2014; Lee \& Chiang 2016; Schlichting 2018). Detailed studies of young exoplanets such as V1298 Tau b may help to answer some of these questions.

We are grateful to Rodrigo Luger, Dan Foreman-Mackey, Marcie Smith, Jeffrey Smith, Konstantin Batygin, Yanqin Wu, and Eve Lee for helpful discussions, and to Scott Davidoff for guidance on figures. This work made use of the gaia-kepler.fun crossmatch database created by Megan Bedell. T.J.D. and E.E.M. gratefully acknowledge support from the Jet Propulsion Laboratory Exoplanetary Science Initiative. E.E.M. acknowledges support from the NASA NExSS program. E.A.P. is supported through a Hubble Fellowship. Part of this research was carried out at the Jet Propulsion Laboratory, California Institute of Technology, under a contract with NASA. This paper includes data collected by the Kepler mission, funded by the NASA Science Mission directorate. This work has made use of data from the European Space Agency (ESA) mission Gaia (https://www.cosmos.esa.int/gaia), processed by the Gaia Data Processing and Analysis Consortium (DPAC, https:// www.cosmos.esa.int/web/gaia/dpac/consortium). Funding for the DPAC has been provided by national institutions, in particular the institutions participating in the Gaia Multilateral Agreement. Some data presented herein were obtained at W.M. Keck Observatory, which is operated as a scientific partnership among the CIT, the Univ. of California and NASA. The authors recognize and acknowledge the significant cultural role and reverence that the summit of Maunakea has always had within the indigenous Hawaiian community. We are most fortunate to conduct observations from this mountain. 
Facilities: Gaia, Keck:I (HIRES), Keck:II (NIRC2), Kepler.

Software: astropy (Astropy Collaboration et al. 2013, 2018), BANYAN $\Sigma$ (Gagné et al. 2018), emcee (ForemanMackey et al. 2013), exoplanet (Foreman-Mackey et al. 2019), EVEREST 2 . 0 (Luger et al. 2018), K2 SC (Aigrain et al. 2016), K2PHOT (Petigura et al. 2018), lightkurve (Barentsen et al. 2019), lmfit (Newville et al. 2014), matplotlib (Hunter 2007), PyMC3 (Salvatier et al. 2016), PyTransit (Parviainen 2015), RadVel (Fulton et al. 2018), starry (Luger et al. 2019), TERRA (Petigura et al. 2013), theano (Theano Development Team 2016).

\section{Appendix A False-positive Scenario Assessment}

An eclipsing binary or a planet transiting a star other than V1298 Tau would be capable of producing the observed transit signal. Below, we assess the likelihood of each possible falsepositive scenario in a quantitative manner when possible and qualitatively otherwise.

We first consider a scenario in which the transit signal is due to an unassociated eclipsing binary widely separated from V1298 Tau on the sky. Occasionally, a bright eclipsing binary within the telescope field of view may contaminate pixels elsewhere on the detector and lead to spurious detections of transiting planets. There are several stars, some saturated, in the vicinity of V1298 Tau on the $K 2$ detector. We examined light curves for each of these stars (EPIC IDs 210819568, 210817793, 210818376) and confirmed that none showed eclipses. We similarly examined light curves for neighboring stars identified using the STAR.NEIGHBORS function with EVEREST 2.0, and found no eclipsing binaries among those stars (EPIC IDs 210787602, 210755820, 210852007, 210832303, 210829398, 210769653, 210828429, 210815489, 210761528).

Our group also performed a systematic search of every $K 2$ light curve from Campaign 4 (Crossfield et al. 2016) for periodic transits or eclipses using the TERRA code (Petigura et al. 2013). We used the output of this search to attempt a cross-match between the ephemeris of V1298 Tau with known periodic signals detected by TERRA above a signal-to-noise of 10. We examined the $K 2$ light curves for 50 stars having signals detected with periods within 0.5 days of the period of V1298 Tau b or half of that period. We found none of those stars to show eclipses coinciding with the transit times of V1298 Tau b. A search of the Kepler Eclipsing Binary Catalog (http://keplerebs.villanova.edu/) for eclipsing binaries discovered from Campaign 4 of the $K 2$ mission also yielded no candidates with period differences of $<0.64$ days relative to V1298 Tau b.

Next we consider closely projected eclipsing binaries that are either associated or unassociated with V1298 Tau. An eclipsing binary can dim by at most $100 \%$. The observed transit depth therefore sets an upper limit to the brightness difference in the Kepler bandpass of 5.7 mag between V1298 Tau and a putative eclipsing binary capable of producing the observed transit depths. The target pixel files for V1298 Tau contain an unassociated star, Gaia DR2 51886331671984640, which resides 19 ". 8 to the southwest. This star is more than 6 mag fainter than V1298 Tau at optical wavelengths, and therefore too faint to be responsible for the periodic transits. Using the interactive LIGHTKURVE ${ }^{18}$ tool, we examined time-series photometry for V1298 Tau from a $24^{\prime \prime} \times 24^{\prime \prime}$ square aperture as well as from a $12^{\prime \prime} \times 8^{\prime \prime}$ rectangular aperture surrounding the fainter source to the southwest. We confirmed that the fainter source is not an eclipsing binary and that the transit signal originates from the region surrounding V1298 Tau.

False-positive scenarios involving hierarchical triples, e.g., a bound eclipsing binary or a planet transiting an undetected companion to V1298 Tau, are similarly disfavored. Nguyen et al. (2012) searched for close companions to V1298 Tau through radial velocity monitoring. Those measurements rule out most scenarios in which V1298 Tau hosts a stellar mass companion with an orbital period between 1 and 100 days. Furthermore, dynamical stability arguments (Mardling \& Aarseth 2001) and empirical evidence from hierarchical multiple star systems (Tokovinin 2018) suggest the ratio of the outer to inner periods in a coplanar hierarchical triple must exceed 4.7. Thus, hierarchical triple scenarios involving a coplanar stellar companion with an orbital period $<113$ days ( $\sim 0.5 \mathrm{au}, \sim 5$ mas) are a priori unlikely.

Although hierarchical triple scenarios are considered unlikely, we consider the implications of such scenarios here. If V1298 Tau is in fact a binary and the transiting body orbits the primary star, the effect of flux dilution is then maximized when the undetected companion is equal in brightness. In such a scenario, the planet's true radius is larger by a factor of $\sqrt{2}$, corresponding to a radius for the transiting companion of $1.3 R_{\text {Jup }}$ (Ciardi et al. 2015). Notably, such a scenario would still favor a planetary nature for the transiting companion given that low-mass stars and brown dwarfs have radii $\gtrsim 2 R_{\text {Jup }}$ at ages $<40 \mathrm{Myr}$. However, if the transiting body instead orbits an undetected companion star, then the radius is most severely underestimated when the optical contrast between a putative companion and V1298 Tau is maximized. Using evolutionary models and previously established methods (David et al. 2016), we calculated the corrected companion radius and mass in such scenarios, finding that the mass of the transiting body could exceed $0.1 M_{\odot}$ if V1298 Tau hosts an undetected companion of $0.5 M_{\odot}$ or lower.

The mean stellar density measured from the transits provides another means of assessing false-positive scenarios. Using the posteriors in period and $a / R_{*}$ from a circular orbit fit, we calculated the mean stellar density and compared it to stellar evolution models in a temperature-density diagram (Figure 21). Although this analysis assumes a circular orbit and should thus be regarded with caution, we find the stellar age implied by the density posterior is consistent with our Hertzsprung-Russell diagram analysis and inconsistent with a main-sequence star or a post-main-sequence star of similar effective temperature and with an age less than the age of the universe.

\footnotetext{
18 http://docs.lightkurve.org
} 

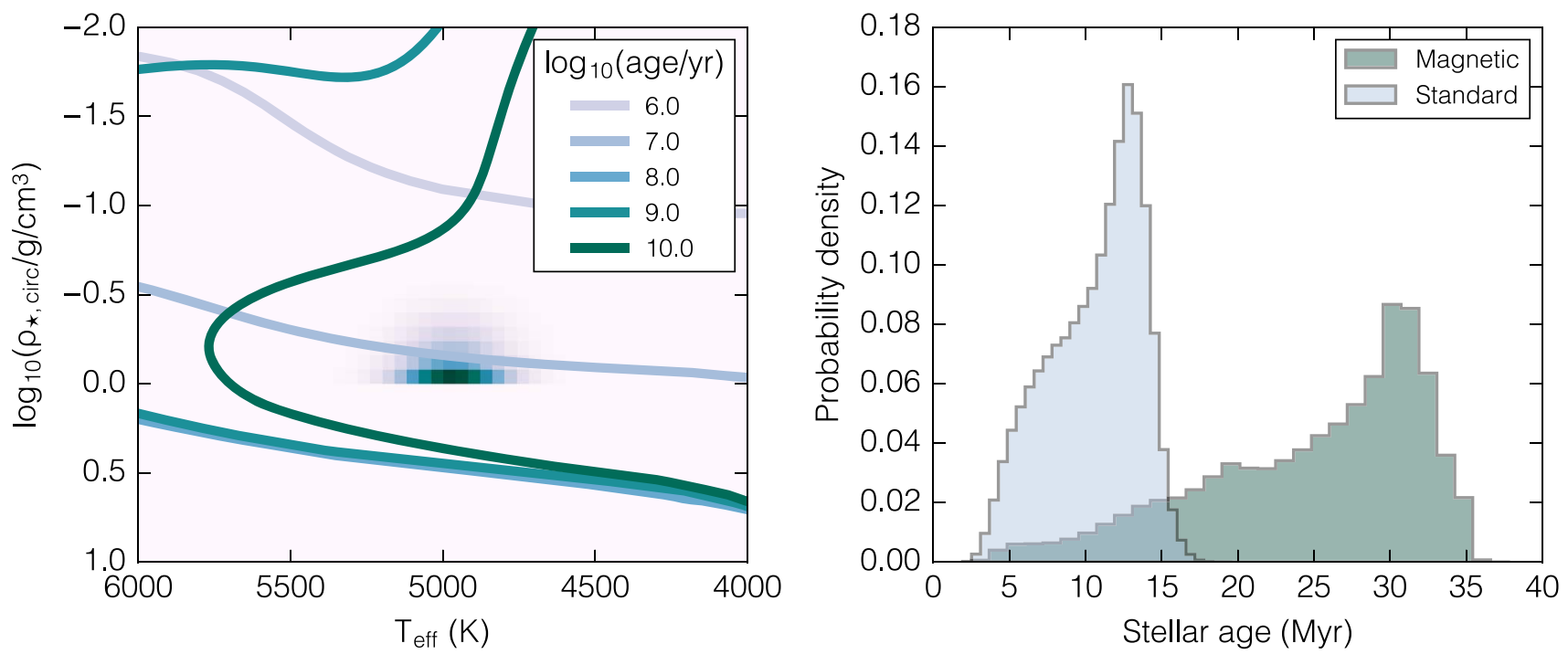

Figure 21. Age inference in a temperature-density diagram. Left: the mean stellar density as a function of effective temperature varies with age (solid lines). The twodimensional histogram shows a normal distribution in temperature and the mean stellar density posterior from the V1298 Tau b transit fit assuming a circular orbit. Right: the probability distributions in age for V1298 Tau resulting from a linear interpolation of the values at left using two different evolutionary models (Dotter et al. 2008; Feiden 2016).

\section{Appendix B \\ Favorability for Follow-up Observations}

We compared V1298 Tau $b$ with the population of confirmed transiting exoplanets using data from the NASA Exoplanet Archive (Akeson et al. 2013), accessed on 2019 May 15. We first compared the favorability for atmospheric characterization between V1298 Tau b and known transiting planets. We followed the methods of Vanderburg et al. (2016) to predict a scaled signal-to-noise for transmission spectroscopy observations using the following equations:

$$
\mathrm{S} / \mathrm{N} \propto \frac{R_{P} H \sqrt{F t_{14}}}{R_{\star}^{2}}
$$

and

$$
H=\frac{k_{\mathrm{B}} T_{\mathrm{eq}}}{\mu g},
$$

where $R_{\mathrm{P}}$ and $R_{\star}$ are the radii of the planet and star, respectively, $H$ is the atmospheric scale height, $F$ is the stellar flux at $H$-band, $t_{14}$ is the total transit duration, $k_{\mathrm{B}}$ is Boltzmann's constant, $T_{\text {eq }}$ is the planet's equilibrium temperature, $\mu$ is the mean molecular weight of the planet's atmosphere, and $g$ is the planet's surface gravity. When available, we used the known mass and radius to calculate surface gravity. Otherwise, we calculated a predicted mass using the planet's radius and an exoplanet mass-radius relation (Weiss et al. 2018). The mean molecular weight was fixed to the Jovian value for planets with radii larger than $1.5 R_{\oplus}$ and to the terrestrial value otherwise.

The scaled transmission spectrum signal-to-noise as a function of $H$-band brightness, planet radius, and equilibrium temperature is depicted in Figure 22. Using this simplistic methodology, we determined that V1298 Tau b ranks among the top 40 most favorable transiting exoplanets for atmospheric characterization. However, in the optimistic case that the planet in fact has a core-dominated composition with a total mass of $30 M_{\oplus}, \mathrm{V} 1298$ Tau b would be in the top five in terms of favorability for infrared transmission spectroscopy using the metrics described. Of course, the unusually high stellar activity presents both challenges (potentially requiring multiple transit observations to disentangle stellar and planetary signals) and opportunities (for studying star-planet interactions).

There are currently 16 known exoplanets with radii $>0.8 R_{\text {Jup }}$, periods between 10 and 50 days, and well-measured masses $<10 M_{\text {Jup }}$. Those planets have masses ranging from 0.2 to $3.5 M_{\text {Jup }}$. For this range of plausible planet masses, the expected radial velocity semi-amplitude ranges from 13 to $230 \mathrm{~m} \mathrm{~s}^{-1}$. If $\mathrm{V} 1298 \mathrm{Tau} \mathrm{b}$ is contracting, implying its presentday radius may be an imprecise indicator of its mass, the true radial velocity amplitude may be lower. We have measured the optical radial velocity jitter to be $\sigma_{\mathrm{RV}}=216,71$, and $5 \mathrm{~m} \mathrm{~s}^{-1}$ over 5.2 days, $10 \mathrm{hr}$, and $30 \mathrm{~min}$, respectively. Measuring the planet's mass may be feasible with newly commissioned infrared spectrographs, due to the lower expected intrinsic stellar variability at redder wavelengths.

V1298 Tau is also a favorable target for measuring the planet's obliquity through the Rossiter-McLaughlin effect or Doppler tomography. Neglecting limb-darkening effects, the Rossiter-McLaughlin amplitude is approximately $A_{\mathrm{RM}} \sim$ $v \sin i \times\left(R_{P} / R_{\star}\right)^{2} \times \sqrt{1-b^{2}}$, which is $117 \mathrm{~m} \mathrm{~s}^{-1}$ for V1298 Tau. Assuming an albedo of 0.3 and neglecting remnant heat from the planet's formation, the predicted secondary eclipse depth is $\delta_{\mathrm{SE}}=\left(R_{\mathrm{P}} / R_{\star}\right)^{2}\left(T_{\mathrm{eq}} / T_{\star}\right) \approx 610 \mathrm{ppm}$, which is detectable with the Spitzer space telescope. A measurement of the temperature of V1298 Tau b could help to constrain planet evolution models. 


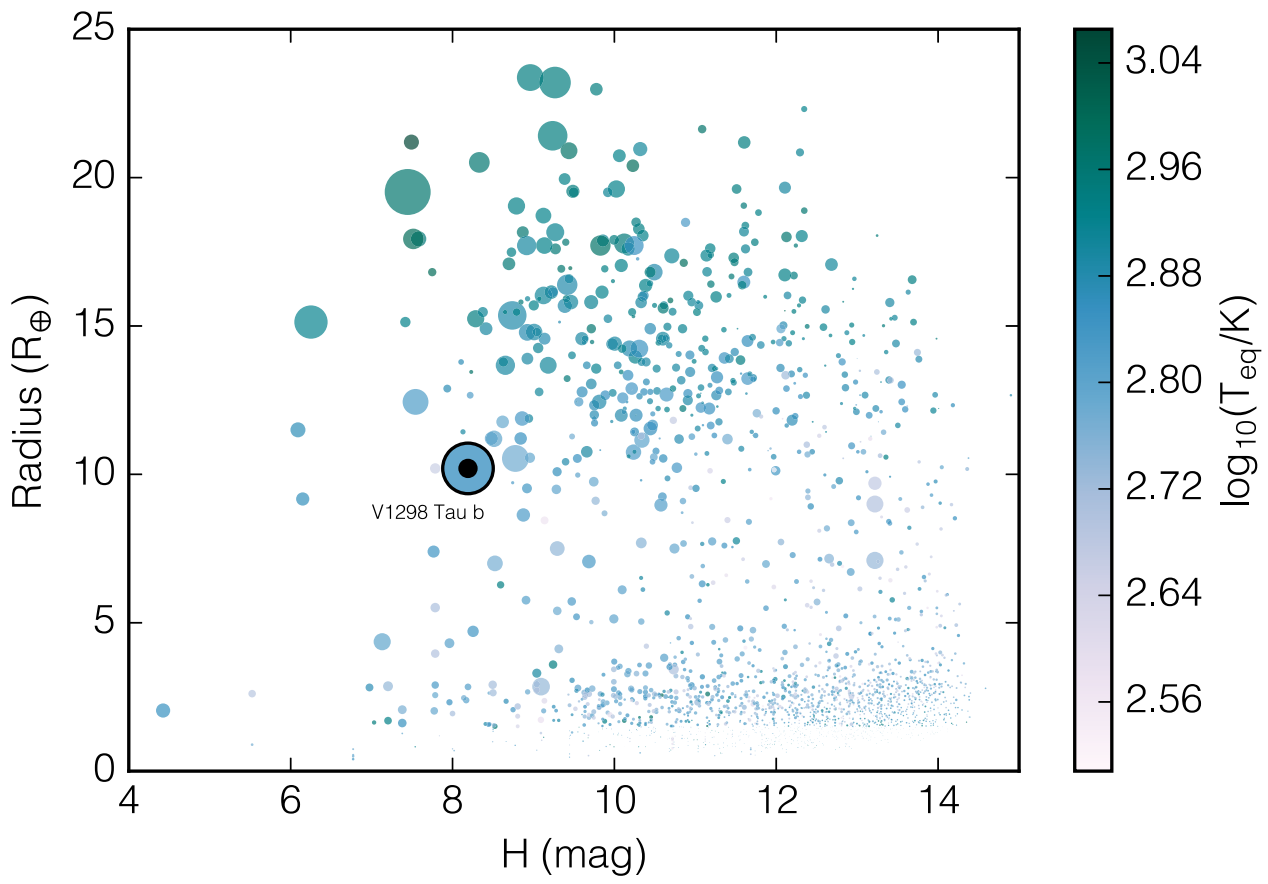

Figure 22. Favorability for transmission spectroscopy; the distribution of known transiting exoplanets as a function of $H$-band brightness and planet radius. The point size is proportional to the scaled signal-to-noise predicted for transmission spectroscopy observations. Point colors indicate the logarithm of the planet equilibrium temperature, assuming an albedo of 0.3 . V1298 Tau b is shown as the point with the black border. The outer boundary represents an optimistic case of $M_{\mathrm{P}}=10 M_{\oplus}$, while the black point at the center indicates the case of $M_{\mathrm{P}}=100 M_{\oplus}$.

\section{ORCID iDs}

Trevor J. David (ib https://orcid.org/0000-0001-6534-6246 Ann Marie Cody (il https://orcid.org/0000-0002-3656-6706 Christina L. Hedges ํㅣ https://orcid.org/0000-0002-3385-8391 Eric E. Mamajek 니 https://orcid.org/0000-0003-2008-1488 Erik A. Petigura (i) https://orcid.org/0000-0003-0967-2893 Benjamin J. Fulton (ib) https://orcid.org/0000-0003-3504-5316 Howard T. Isaacson 즈 https://orcid.org/0000-0002-0531-1073 Andrew W. Howard (1) https://orcid.org/0000-00018638-0320

Jonathan Gagné (i] https://orcid.org/0000-0002-2592-9612 Luisa M. Rebull (i) https://orcid.org/0000-0001-6381-515X John R. Stauffer (i) https://orcid.org/0000-0003-3595-7382 Sasha Hinkley @ https://orcid.org/0000-0001-8074-2562

\section{References}

Aigrain, S., Parviainen, H., \& Pope, B. J. S. 2016, MNRAS, 459, 2408

Akeson, R. L., Chen, X., Ciardi, D., et al. 2013, PASP, 125, 989

Anderson, K. R., \& Lai, D. 2017, MNRAS, 472, 3692

Antonini, F., Hamers, A. S., \& Lithwick, Y. 2016, AJ, 152, 174

Astropy Collaboration, Price-Whelan, A. M., Sipőcz, B. M., et al. 2018, AJ, 156,123

Astropy Collaboration, Robitaille, T. P., Tollerud, E. J., et al. 2013, A\&A, 558, A33

Baraffe, I., Chabrier, G., Barman, T. S., Allard, F., \& Hauschildt, P. H. 2003, A\&A, 402, 701

Baraffe, I., Homeier, D., Allard, F., \& Chabrier, G. 2015, A\&A, 577, A42

Barentsen, G., Hedges, C., Vinícius, Z., et al. 2019, KeplerGO/lightkurve: Lightkurve v1.0b29., Zenodo, doi:10.5281/zenodo.2565212

Barrado y Navascués, D., Deliyannis, C. P., \& Stauffer, J. R 2001, ApJ, 549,452

Batygin, K., Bodenheimer, P. H., \& Laughlin, G. P. 2016, ApJ, 829, 114

Binks, A. S., \& Jeffries, R. D. 2014, MNRAS, 438, L11

Boley, A. C., Granados Contreras, A. P., \& Gladman, B. 2016, ApJL, 817, L17

Boller, T., Freyberg, M. J., Trümper, J., et al. 2016, A\&A, 588, A103

Bouvier, J., Barrado, D., Moraux, E., et al. 2018, A\&A, 613, A63

Bowler, B. P. 2016, PASP, 128, 102001
Burrows, A., Marley, M., Hubbard, W. B., et al. 1997, ApJ, 491, 856 Capitanio, L., Lallement, R., Vergely, J. L., Elyajouri, M., \& Monreal-Ibero, A. 2017, A\&A, 606, A65

Chen, H., \& Rogers, L. A. 2016, ApJ, 831, 180

Ciardi, D. R., Beichman, C. A., Horch, E. P., \& Howell, S. B. 2015, ApJ, 805,16

Ciardi, D. R., Crossfield, I. J. M., Feinstein, A. D., et al. 2018, AJ, 155, 10

Claret, A., Hauschildt, P. H., \& Witte, S. 2012, A\&A, 546, A14

Claret, A., Hauschildt, P. H., \& Witte, S. 2013, A\&A, 552, A16

Crossfield, I. J. M., Ciardi, D. R., Petigura, E. A., et al. 2016, ApJS, 226, 7

Curtis, J. L., Vanderburg, A., Torres, G., et al. 2018, AJ, 155, 173

Daemgen, S., Bonavita, M., Jayawardhana, R., Lafrenière, D., \& Janson, M. 2015, ApJ, 799, 155

David, T. J., Hillenbrand, L. A., Petigura, E. A., et al. 2016, Natur, 534, 658

Davies, C. L., Gregory, S. G., \& Greaves, J. S. 2014, MNRAS, 444, 1157

Dawson, R. I., \& Chiang, E. 2014, Sci, 346, 212

Dawson, R. I., \& Johnson, J. A. 2012, ApJ, 756, 122

Dawson, R. I., Murray-Clay, R. A., \& Johnson, J. A. 2015, ApJ, 798, 66

Deming, D., Wilkins, A., McCullough, P., et al. 2013, ApJ, 774, 95

Dobashi, K., Uehara, H., Kandori, R., et al. 2005, PASJ, 57, S1

Donati, J. F., Moutou, C., Malo, L., et al. 2016, Natur, 534, 662

Dong, S., Katz, B., \& Socrates, A. 2014, ApJL, 781, L5

Dotter, A., Chaboyer, B., Jevremović, D., et al. 2008, ApJS, 178, 89

Espinoza, N. 2018, RNAAS, 2, 209

Evans, D. F. 2018, RNAAS, 2, 20

Feiden, G. A. 2016, A\&A, 593, A99

Findeisen, K., \& Hillenbrand, L. 2010, AJ, 139, 1338

Foreman-Mackey, D., Barentsen, G., \& Barclay, T. 2019, dfm/exoplanet: exoplanet v0.1.5., Zenodo, doi:10.5281/zenodo. 2587222

Foreman-Mackey, D., Hogg, D. W., Lang, D., \& Goodman, J. 2013, PASP, 125,306

Fortney, J. J., Marley, M. S., \& Barnes, J. W. 2007, ApJ, 659, 1661

Fortney, J. J., \& Nettelmann, N. 2010, SSRv, 152, 423

Fulton, B. J., \& Petigura, E. A. 2018, AJ, 156, 264

Fulton, B. J., Petigura, E. A., Blunt, S., \& Sinukoff, E. 2018, PASP, 130, 044504

Fulton, B. J., Petigura, E. A., Howard, A. W., et al. 2017, AJ, 154, 109

Furlan, E., Ciardi, D. R., Everett, M. E., et al. 2017, AJ, 153, 71

Gagné, J., Mamajek, E. E., Malo, L., et al. 2018, ApJ, 856, 23

Gaia Collaboration, Brown, A. G. A., Vallenari, A., et al. 2018, A\&A, 616, A1

Gelman, A., \& Rubin, D. B. 1992, StaSc, 7, 457

Ginzburg, S., Schlichting, H. E., \& Sari, R. 2018, MNRAS, 476, 759 
Girardi, L., Barbieri, M., Groenewegen, M. A. T., et al. 2012, ASSP, 26, 165 Goodman, J., \& Weare, J. 2010, CAMCOS, 5, 65

Grankin, K. N., Artemenko, S. A., \& Melnikov, S. Y. 2007, IBVS, 5752, 1

Gray, D. F. 2005, The Observation and Analysis of Stellar Photospheres (3rd ed.; Cambridge: Cambridge Univ. Press)

Gupta, A., \& Schlichting, H. E. 2019, MNRAS, 487, 24

Hansen, B. M. S., \& Murray, N. 2012, ApJ, 751, 158

Hartmann, L., Jones, B. F., Stauffer, J. R., \& Kenyon, S. J. 1991, AJ, 101, 1050 Hoffman, M. D., \& Gelman, A. 2014, JMLR, 15, 1593

Howard, A. W., Johnson, J. A., Marcy, G. W., et al. 2010a, ApJ, 721, 1467

Howard, A. W., Marcy, G. W., Johnson, J. A., et al. 2010b, Sci, 330, 653

Howell, S. B., Sobeck, C., Haas, M., et al. 2014, PASP, 126, 398

Huang, C., Wu, Y., \& Triaud, A. H. M. J. 2016, ApJ, 825, 98

Hunter, J. D. 2007, CSE, 9, 90

Ida, S., \& Lin, D. N. C. 2010, ApJ, 719, 810

Jeffries, R. D., Naylor, T., Mayne, N. J., Bell, C. P. M., \& Littlefair, S. P. 2013, MNRAS, 434, 2438

Jin, S., Mordasini, C., Parmentier, V., et al. 2014, ApJ, 795, 65

Johns-Krull, C. M., McLane, J. N., Prato, L., et al. 2016, ApJ, 826, 206

Jones, B. F., Shetrone, M., Fischer, D., \& Soderblom, D. R. 1996, AJ, 112, 186

Kipping, D. M. 2010, MNRAS, 407, 301

Kipping, D. M. 2013a, MNRAS, 435, 2152

Kipping, D. M. 2013b, MNRAS, 434, L51

Kley, W., \& Nelson, R. P. 2012, ARA\&A, 50, 211

Kolbl, R., Marcy, G. W., Isaacson, H., \& Howard, A. W. 2015, AJ, 149, 18

Kraus, A. L., Herczeg, G. J., Rizzuto, A. C., et al. 2017, ApJ, 838, 150

Kraus, A. L., Shkolnik, E. L., Allers, K. N., \& Liu, M. C. 2014, AJ, 147, 146

Lallement, R., Vergely, J.-L., Valette, B., et al. 2014, A\&A, 561, A91

Lee, E. J., \& Chiang, E. 2016, ApJ, 817, 90

Lee, E. J., Chiang, E., \& Ormel, C. W. 2014, ApJ, 797, 95

Libralato, M., Nardiello, D., Bedin, L. R., et al. 2016, MNRAS, 463, 1780

Livingston, J. H., Dai, F., Hirano, T., et al. 2018, AJ, 155, 115

Livingston, J. H., Dai, F., Hirano, T., et al. 2019, MNRAS, 484, 8

Lomb, N. R. 1976, Ap\&SS, 39, 447

Lopez, E. D., \& Fortney, J. J. 2013, ApJ, 776, 2

Luger, R., Agol, E., Foreman-Mackey, D., et al. 2019, AJ, 157, 64

Luger, R., Kruse, E., Foreman-Mackey, D., Agol, E., \& Saunders, N. 2018, AJ, 156,99

Luhman, K. L. 2018, arXiv:1811.01359

Malo, L., Doyon, R., Lafrenière, D., et al. 2013, ApJ, 762, 88

Mamajek, E. E., \& Hillenbrand, L. A. 2008, ApJ, 687, 1264

Mandel, K., \& Agol, E. 2002, ApJL, 580, L171

Mann, A. W., Gaidos, E., Mace, G. N., et al. 2016a, ApJ, 818, 46

Mann, A. W., Gaidos, E., Vanderburg, A., et al. 2017, AJ, 153, 64

Mann, A. W., Newton, E. R., Rizzuto, A. C., et al. 2016b, AJ, 152, 61

Mann, A. W., Vanderburg, A., Rizzuto, A. C., et al. 2018, AJ, 155, 4

Mardling, R. A., \& Aarseth, S. J. 2001, MNRAS, 321, 398

Marley, M. S., Fortney, J. J., Hubickyj, O., Bodenheimer, P., \& Lissauer, J. J. 2007, ApJ, 655, 541

Martin, D. C., Fanson, J., Schiminovich, D., et al. 2005, ApJL, 619, L1

Mayor, M., Marmier, M., Lovis, C., et al. 2011, arXiv:1109.2497

Mentuch, E., Brandeker, A., van Kerkwijk, M. H., Jayawardhana, R., \& Hauschildt, P. H. 2008, ApJ, 689, 1127

Mordasini, C. 2013, A\&A, 558, A113

Mordasini, C., Alibert, Y., Georgy, C., et al. 2012, A\&A, 547, A112

Mordasini, C., Marleau, G.-D., \& Mollière, P. 2017, A\&A, 608, A72

Newville, M., Stensitzki, T., Allen, D. B., \& Ingargiola, A. 2014, LMFIT: NonLinear Least-Square Minimization and Curve-Fitting for Python, Zenodo, doi:10.5281/zenodo.11813

Nguyen, D. C., Brandeker, A., van Kerkwijk, M. H., \& Jayawardhana, R. 2012, ApJ, 745, 119
Nidever, D. L., Marcy, G. W., Butler, R. P., Fischer, D. A., \& Vogt, S. S. 2002, ApJS, 141, 503

Obermeier, C., Henning, T., Schlieder, J. E., et al. 2016, AJ, 152, 223

Oh, S., Price-Whelan, A. M., Hogg, D. W., Morton, T. D., \& Spergel, D. N. 2017, AJ, 153, 257

Olmedo, M., Lloyd, J., Mamajek, E. E., et al. 2015, ApJ, 813, 100

Owen, J. E., \& Wu, Y. 2013, ApJ, 775, 105

Palla, F., \& Stahler, S. W. 2002, ApJ, 581, 1194

Parviainen, H. 2015, MNRAS, 450, 3233

Pecaut, M. J., \& Mamajek, E. E. 2013, ApJS, 208, 9

Pepper, J., Gillen, E., Parviainen, H., et al. 2017, AJ, 153, 177

Petigura, E. A., Crossfield, I. J. M., Isaacson, H., et al. 2018, AJ, 155, 21

Petigura, E. A., Howard, A. W., \& Marcy, G. W. 2013, PNAS, 110, 19273

Petigura, E. A., Sinukoff, E., Lopez, E. D., et al. 2017, AJ, 153, 142

Petrovich, C., \& Tremaine, S. 2016, ApJ, 829, 132

Quinn, S. N., White, R. J., Latham, D. W., et al. 2012, ApJL, 756, L33

Quinn, S. N., White, R. J., Latham, D. W., et al. 2014, ApJ, 787, 27

Randich, S., Pallavicini, R., Meola, G., Stauffer, J. R., \& Balachandran, S. C. 2001, A\&A, 372, 862

Rebull, L. M., Stauffer, J. R., Bouvier, J., et al. 2016, AJ, 152, 113

Rebull, L. M., Stauffer, J. R., Cody, A. M., et al. 2018, AJ, 155, 196

Rizzuto, A. C., Mann, A. W., Vanderburg, A., Kraus, A. L., \& Covey, K. R. 2017, AJ, 154, 224

Rizzuto, A. C., Vanderburg, A., Mann, A. W., et al. 2018, AJ, 156, 195

Salvatier, J., Wiecki, T. V., \& Fonnesbeck, C. 2016, PeerJ Computer Science, 2, e55

Santerne, A., Moutou, C., Tsantaki, M., et al. 2016, A\&A, 587, A64

Scargle, J. D. 1982, ApJ, 263, 835

Schlichting, H. E. 2018, in Handbook of Exoplanets, ed. H. J. Deeg \& J. A. Belmonte (Cham: Springer), 141

Schlichting, H. E., Sari, R., \& Yalinewich, A. 2015, Icar, 247, 81

Skrutskie, M. F., Cutri, R. M., Stiening, R., et al. 2006, AJ, 131, 1163

Slesnick, C. L., Carpenter, J. M., Hillenbrand, L. A., \& Mamajek, E. E. 2006, AJ, 132, 2665

Smith, J. C., Stumpe, M. C., Van Cleve, J. E., et al. 2012, PASP, 124, 1000

Soderblom, D. R., Jones, B. F., Balachandran, S., et al. 1993, AJ, 106, 1059

Spiegel, D. S., \& Burrows, A. 2012, ApJ, 745, 174

Stumpe, M. C., Smith, J. C., Van Cleve, J. E., et al. 2012, PASP, 124, 985

Terquem, C., \& Papaloizou, J. C. B. 2007, ApJ, 654, 1110

Theano Development Team 2016, arXiv: 1605.02688

Thompson, S. E., Coughlin, J. L., Hoffman, K., et al. 2018, ApJS, 235, 38

Tokovinin, A. 2018, ApJS, 235, 6

Udry, S., Mayor, M., \& Santos, N. C. 2003, A\&A, 407, 369

van Eyken, J. C., Ciardi, D. R., von Braun, K., et al. 2012, ApJ, 755, 42

Vanderburg, A., Bieryla, A., Duev, D. A., et al. 2016, ApJL, 829, L9

Vanderburg, A., Mann, A. W., Rizzuto, A., et al. 2018, AJ, 156, 46

Vogt, S. S., Allen, S. L., Bigelow, B. C., et al. 1994, Proc. SPIE, 2198, 362

Wahhaj, Z., Cieza, L., Koerner, D. W., et al. 2010, ApJ, 724, 835

Waite, I. A., Marsden, S. C., Carter, B. D., et al. 2011, MNRAS, 413, 1949

Weiss, L. M., Marcy, G. W., Petigura, E. A., et al. 2018, AJ, 155, 48

Wichmann, R., Krautter, J., Schmitt, J. H. M. M., et al. 1996, A\&A, 312, 439

Wichmann, R., Torres, G., Melo, C. H. F., et al. 2000, A\&A, 359, 181

Wright, N. J., Drake, J. J., Mamajek, E. E., \& Henry, G. W. 2011, ApJ, 743, 48

Yee, J. C., \& Jensen, E. L. N. 2010, ApJ, 711, 303

Yu, L., Donati, J.-F., Hébrard, E. M., et al. 2017, MNRAS, 467, 1342

Yu, L., Winn, J. N., Gillon, M., et al. 2015, ApJ, 812, 48

Yuan, H. B., Liu, X. W., \& Xiang, M. S. 2013, MNRAS, 430, 2188

Zhang, Z., Liu, M. C., Best, W. M. J., et al. 2018, ApJ, 858, 41

Ziegler, C., Law, N. M., Baranec, C., et al. 2018, AJ, 156, 259 